\title{
Experimental and numerical determination of the wave dispersion characteristics of complex 3D woven composites
}

\author{
V. Thierry ${ }^{1}$, O. Mesnil ${ }^{2}$, D.Chronopoulos ${ }^{1}$ \\ ${ }^{1}$ Institute for Aerospace Technology \& The Composites Research Group, The University of Nottingham, NG7 2RD, UK \\ ${ }^{2}$ CEA-List, NDT department, 91191 Gif-Sur-Yvette, France
}

\begin{abstract}
In this article, an attempt is made towards the experimental validation of a numerical method allowing vibro-acoustic and ultrasonic wave propagation analysis of complex woven composites. For this validation, three different woven architectures were used to manufacture composite samples using resin transfer molding process. For modelling purposes, these composites can be considered as periodic structures composed of unit cells repeated in two directions. The unit cells of these three different woven patterns are modelled realistically at a mesoscopic scale, using information from micrographic cross-sections and the mechanical properties of the fibres and matrix materials provided by the manufacturers and the literature. The numerical method, combining the mode-based component mode synthesis and the wave finite element methods, allows for computing the dispersion relations of the modelled samples. Experimentally, dispersion relations are extracted from the samples by means of a linear scan of a wave measured by a laser vibrometer and generated by a piezoelectric transducer. The modelling methodology is thus validated experimentally and shows a good accuracy for determining the dispersion relations of complex woven composites. It is feasible computationally and provides a deeper understanding of the dispersion relations than experimental testing.
\end{abstract}

Keywords: Dispersion relations, 3D woven composites, 2D fast Fourier transform, Component Mode Synthesis, Periodic Structure Theory, Wave propagation, Multiscale modelling

\section{Introduction}

The proportion of composite materials used in the industry is constantly increasing. They are preferred for some specific load carrying elements in the aerospace industry for their numerous advantages such as lightweight and excellent mechanical properties when compared to traditional materials such as aluminium alloys. Even though they are costly, weight saving is critical in this industry as it leads to a reduced fuel consumption and thus to fleet-wise savings. The interest for 3D woven composites over 2D laminates lies in their high resistance in out-of-plane loading and impact where 2D laminates are easily subjected to delamination, with a low speed impact from a dropped tool or high speed hail impact for example $[1,2,3]$. Another advantage is that complex components are easier to manufacture using a 3D reinforcement than stacking multiple thin layers of laminates [2].

Even though more resilient, damage may still occur in $3 \mathrm{D}$ composites and this is the reason why it needs to 
be monitored. Many Non Destructive Evaluation (NDE) methods have been developed and ultrasonic guided wave techniques in particular are widely used in that context. This set of techniques is of great interest because they can be extended to a Structural Health Monitoring (SHM) method thanks to the advantages of the hardware it requires (piezoelectric transducers (PZT) are lightweight, inexpensive and a network of sensors can easily be assembled).

Guided waves can propagate on a long distance in thin waveguides and are sensitive to defects. For these reasons guided waves inspection techniques are commonly used in traditional beam and plate-like structures. Numerous analytical methods simulating Lamb waves and semi-analytical methods such as the Semi-Analytical Finite Element (SAFE) method or the Wave Finite Element Method (WFEM) have been developed for this purpose. While the SAFE method is very time efficient when investigating a material that is inhomogeneous in its thickness but homogeneous in the direction of propagation (such as composite laminates), it encounters severe limitations when it comes to materials that are periodic in the directions of propagation (such as metamaterial or textile composites) [4]. Indeed, in strongly anisotropic composites, such as woven composites, the wave dispersion characteristics are not straightforward to obtain. Many researchers consider these composites as orthotropic materials after homogenising their mechanical properties so they can be considered as laminates but in reality they are not and need to be considered at a mesoscopic scale $[1,5,6,7,8]$. The WFEM is an efficient method when it comes to simulating wave propagation in periodic structures and is the method used in this paper for the numerical simulation.

The principal novelty of this paper is the numerical and experimental determination of the dispersion relations of strongly anisotropic woven composites. The methodology developed in a previous publication [8] while having already been numerically verified, will be experimentally validated. Whereas the numerical models presented in [8] were analytical, this paper emphasises the accurate modelling of actual manufactured composites.

The paper is organised as follows. In Sec.2.1 an overview of the samples manufacturing process is presented. In the following section (Sec.2.2), the method for experimental determination of the dispersion curves is shown. In Sec.3 the multiscale geometric modelling of the composite samples is depicted (three levels of modelling are presented). The following section (Sec.4) presents two numerical methods for determining the dispersion relations in the modeled composites. Finally, results for the three woven composites are shown (Sec.5) and the comparison between the experimental and numerical methods is discussed.

\section{Experimental determination of the wave dispersion properties in 3D woven composites}

For this study, complex woven composite samples have been manufactured and tested. The first subsection gives details on their architectures and on the manufacturing process used. The subsequent subsection explains how the samples were tested to obtain the dispersion characteristics.

\subsection{Manufacturing of the $3 D$ woven composite samples}

Six samples composed of a total of three different architecture reinforcements [9] were manufactured for this study. Their architectures are roughly described here, but further illustrated in Sec.3.2 (in Figs.7-8) as the details are 
of the upmost importance when it comes to geometrical modelling. Definitions of the nomenclature relative to 3D woven textile can be found in [10].

- Three 250x250x1.97 mm plates using a classic through-thickness angle interlock weave architecture reinforcement (Fabric 1), all of them infused with IN2 epoxy resin. Fabric 1 comprises two layers of $2 \times 12 \mathrm{~K}$ tows in the warp direction; three layers of $12 \mathrm{~K}$ tows in the weft direction and $6 \mathrm{~K}$ binder tows, alternating with the warp tows.

- Two 250x250x1.95 mm plates using a less conventional angle interlock weave architecture reinforcement (Fabric 2), all of them infused with the Gurit20 epoxy resin. Fabric 2 comprises two layers of $4 \times 12 \mathrm{~K}$ tows in the warp direction; three layers of $12 \mathrm{~K}$ tows in the weft direction; $6 \mathrm{~K}$ binder tows, alternating with the warp tows and $6 \mathrm{~K}$ tows in the warp direction, interwoven with weft layers on the surfaces of the fabric.

- One 250x250x4.98 mm plate using an orthogonal weave architecture reinforcement (Fabric 3), infused with the IN2 epoxy resin for the matrix. Fabric 3 comprises six layers of $12 \mathrm{~K}$ tows in the warp direction; seven layers of $2 \times 6 \mathrm{~K}$ tows in the weft direction; $1 \mathrm{~K}$ binder tows, alternating with the warp tows.

A through-thickness angle interlock weave architecture reinforcement means the binder yarns travel in a fixed pattern (at a certain angle) from top to bottom. An orthogonal weave architecture reinforcement means the binder yarns are either at top or bottom travelling vertically through layers.

Liquid Composite Moulding (LCM) comprises all the composite manufacturing processes that involves the injection of a liquid resin into a dry fiber preform. There exist various LCM technologies, among them the Resin Transfer Moulding (RTM), Vacuum-Assisted RTM (VARTM), Vacuum Infusion (VI) etc. The RTM technique was chosen above the others because it is consistent, easy to control, it allows for a high rate of production and most importantly for having two smooth sides. The VI and RTM techniques respectively create a sample with one or two smooth and glossy sides. At least one smooth side is necessary as it will be the surface of reflection for the laser vibrometer used for the experimental measurements. Two smooth sides are preferred as it simplifies the geometry of the composite and thus the geometrical modelling. RTM uses a closed metallic mould. A metallic frame, whose thickness will give the final thickness of the samples ( $2 \mathrm{~mm}$ and $5 \mathrm{~mm}$ in our cases), is placed in between the two sealing parts of the mould, each having a flat surface on one side. The $250 \times 250 \mathrm{~mm}$ piece of the preform is placed in the frame as depicted in Fig.1. A limitation of this manufacturing technique is that the dimension of the plates is defined by the frame and mould sizes which in our case are rather modest.

The mould is closed, sealed and four outlet located at each corner of the square mould are placed under vacuum as depicted in Fig.2. The homogeneous resin mixture is degassed by a vacuum pump and placed inside the resin injector. The resin is then transferred from the resin injector to the mould through the central inlet. The resin will flow from the center of the mould to the four corner outlets, filling the internal space of the mould. It takes about 30 minutes for the resin to fill all the free spaces and to impregnate the fibers deeply. The four outlets are connected to a vacuum pot 


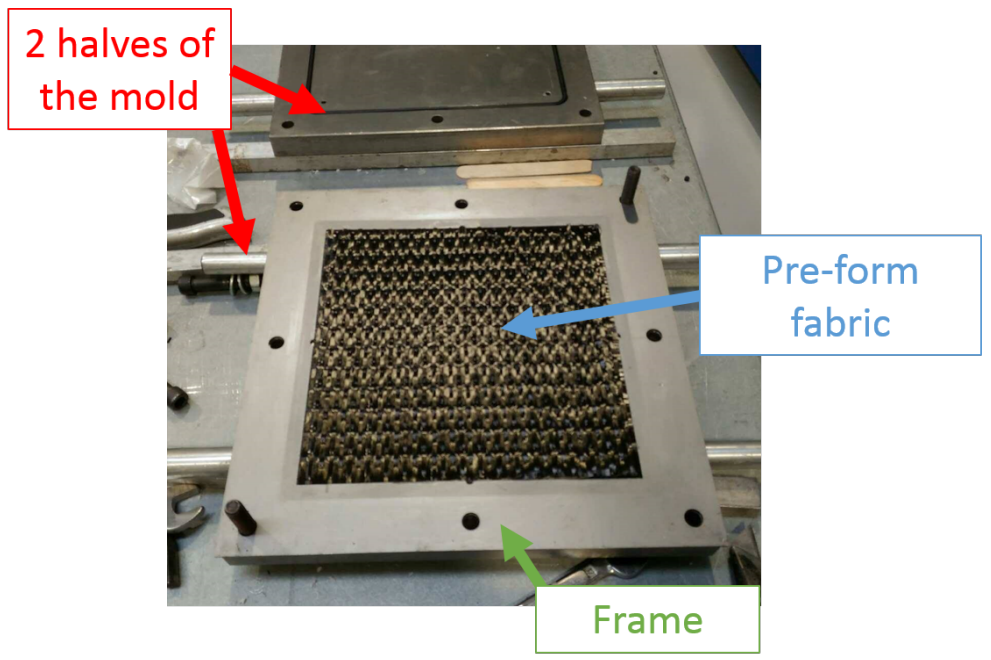

Figure 1: Photography showing the two halves of the mould, with the frame placed on one half and the pre-form fabric sheet fitted inside the frame

to make sure all the air is sucked out of the mould. The resin starts curing after about an hour, depending on the resin used. It is left to cure at room temperature for 24 hours and then post-cured in an oven for about 6 hours, once again depending on the resin used.

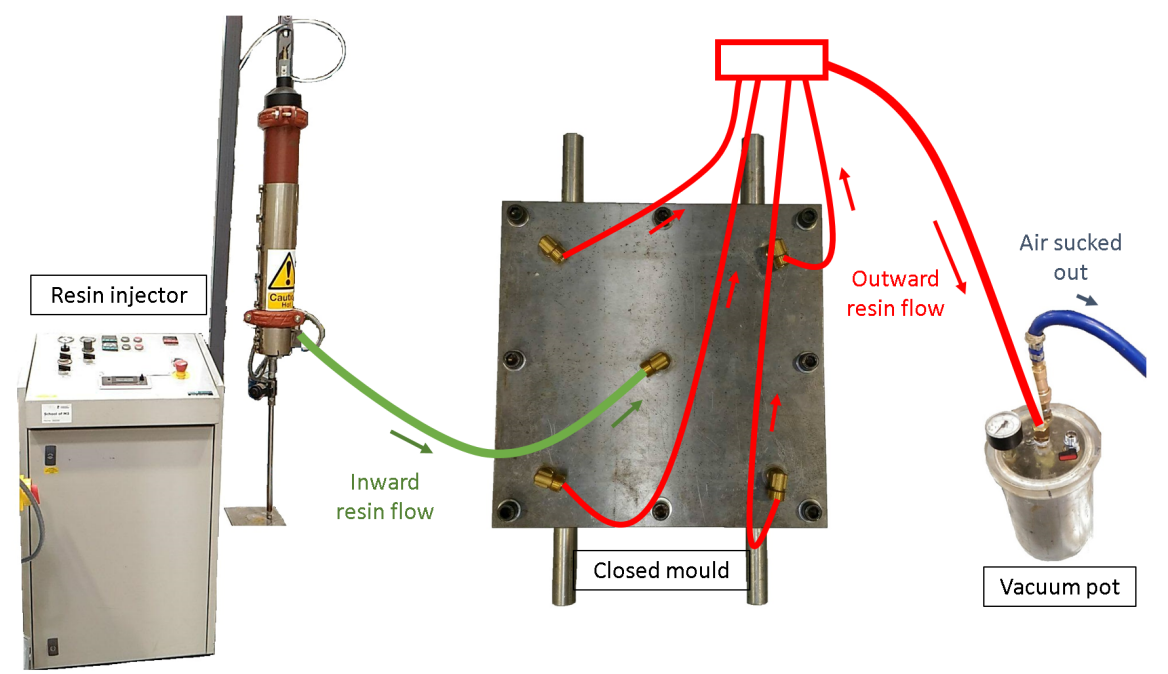

Figure 2: Picture depicting the resin flow from the injector to the sealed mould. The resin in injected from the resin injector to the closed mould through the central inlet. A vacuum is created in the vacuum pot. As the air is sucked out from the mould through the four corner outlets, the resin flows from the center of the mould to the corner exits and fills the mould's free space of resin. 
In order to proceed to a validation of the methodology, several samples composed of the same preform and same resin were manufactured and tested to ensure they provided the same results.

\subsection{Experimental determination of the dispersion curves}

Three steps are necessary in order to experimentally obtain the dispersion curves in the plate samples. Firstly, a piezoeletric transducer is glued to the plate. It is used to generate the guided waves in the plate. Secondly, a laser scan is performed on the plate in order to measure the displacement as a function of the time at different discrete positions on the plate. At last, these measurements are post-processed in order to obtain the results in the frequencywavenumber domain corresponding to the dispersion curves. These steps are further described in the next paragraphs. A broadband excitation signal is transmitted to the studied structure (woven composite plates in our case) by means of a PZT bounded to it. The input excitation signal is sinusoidal periodic and carries a wide frequency band. Two cycles of the sinusoidal function are modulated by a window function (Hanning window here). The interest in having a wide frequency band in the excitation signal, is to obtain the dispersion relations for the same frequency band, performing only one linear scan per direction of interest.

The set-up (see Fig.3) is composed of a waveform generator that is used to generate the electrical waveform transmitted to the piezoelectric transducer. Before it is transmitted to the PZT transducer, the signal is amplified as the waveform generator can only provide a tension of $10 \mathrm{Vpp}$ maximum. The high speed bipolar amplifier allows for reaching a higher tension $(70 \mathrm{Vpp})$ which then allows the signal to propagate further and with a higher amplitude.

The transducer (13 $\mathrm{mm}$ diameter) is glued on the surface of the tested textile composite sample. The Laser Doppler Vibrometer (LDV) is programmed to measure the displacement at a set of discrete positions on a straight line (B-scan) originated at the PZT transducer. The PZT element has to be placed in the continuity of that line. One B-scan provides the direction of propagation along its direction, therefore multiple B-scans of various orientations are required to capture the anisotropic behavior of the materials. The laser vibrometer measures the out-of-plane displacement $\left(U_{3}\right)$ or velocity component which greatly limits the ability to measure the S0 mode.

For each position, the amplitude is measured 200 times with a repetition every $20 \mathrm{~ms}$. The scanning head is then moved to the next discrete position and is connected to a pass band filter which filters high frequency noise and the low frequency vibrations.

This output signal is transmitted to the oscilloscope. The sampling frequency is of 1 Msamples/s. For illustration purposes, a B-scan, i.e. the out-of-plane displacement as measured by the laser as a function of time, is shown in Fig.4.a. The progress and dispersion of the forward going wavepacket can be observed as well as some reflected waves. The almost vertical line, whose magnitude is weak, on the left shows the pressure mode (S0) that is the fastest to propagate, while the second line with a smaller slope and a higher magnitude shows the flexural mode (A0). The flexural mode is more clearly observed as the laser measures the out-of-plane component which is strongly solicited by this mode. 


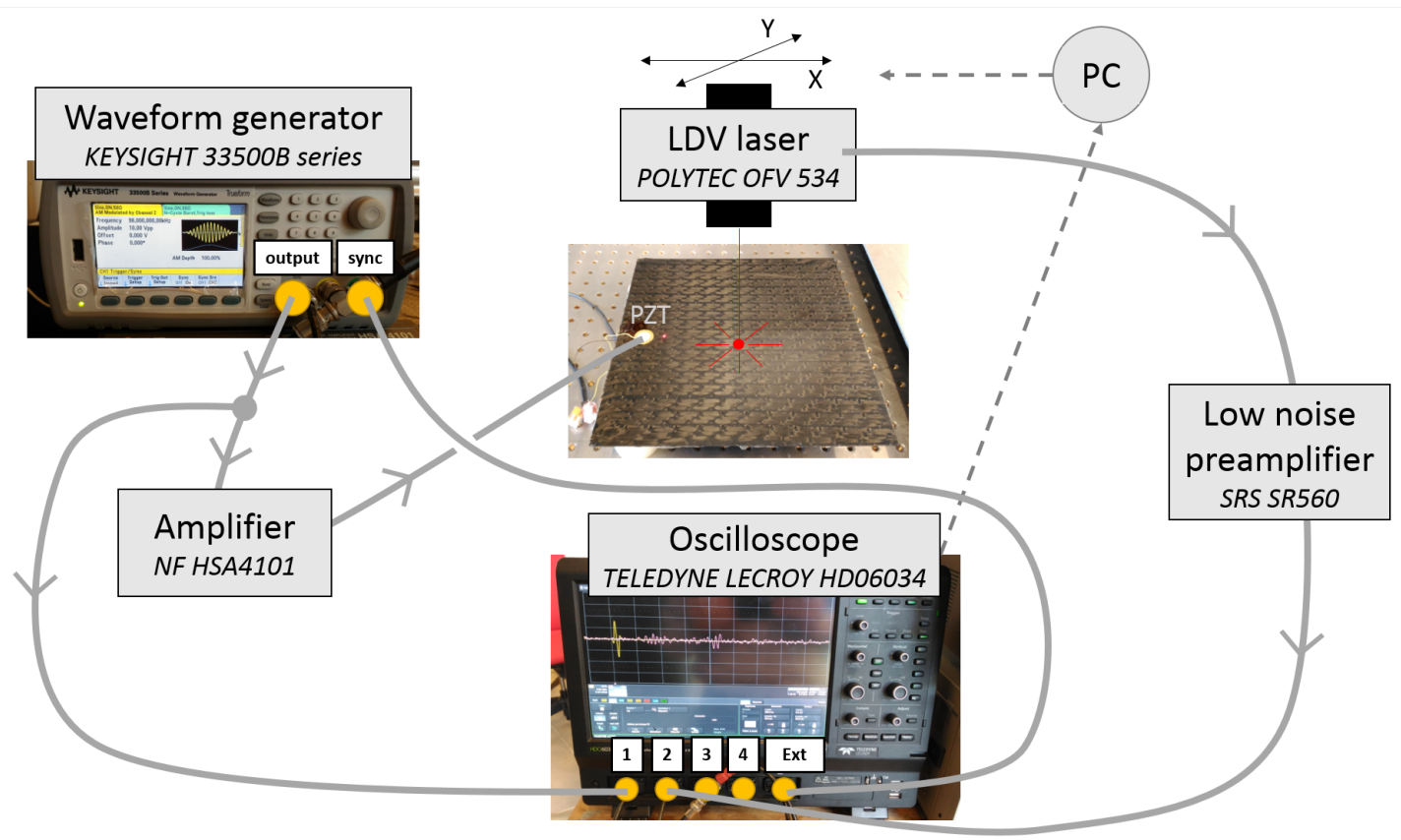

Figure 3: Experimental set-up for B-scan measurements. A signal is generated by the waveform generator, amplified and transmitted to the PZT. The PZT transmits the vibrations to the composite plate. The LDV laser measures the outof-plane displacements which are filtered by the low noise preamplifier. The input and measured signals are recorder by the oscilloscope.

One of the difficulty associated with the measurement of the dispersion relations in a thin structure (beam or plate for example) is that at least two modes can exist and propagate for any given frequency. The two-dimensional fast Fourier transform (2D FFT) [11, 12] is applied to obtain the dispersion curves.

In the dispersion curves of Fig.4.b, the A0 mode can be clearly observed, while the S0 mode is barely visible. To highlight the visibility of the S0 mode, an angle of 15 degrees (relatively to the normal and around the y-axis) was given to the laser beam so that a small portion of the $U_{1}$ component could be captured as well, and $\mathrm{S} 0$ observed. Also, the resolution on the figure is of poor quality, this is explained by the rather small size of the sample plates limited by the manufacturing equipment used. Indeed, a pixel height (in the wavenumber direction) is inversely proportional to the scanned length. In our case, the small plate length $(250 \times 250 \mathrm{~mm})$ results in large pixels and thus a low wavenumber resolution. 


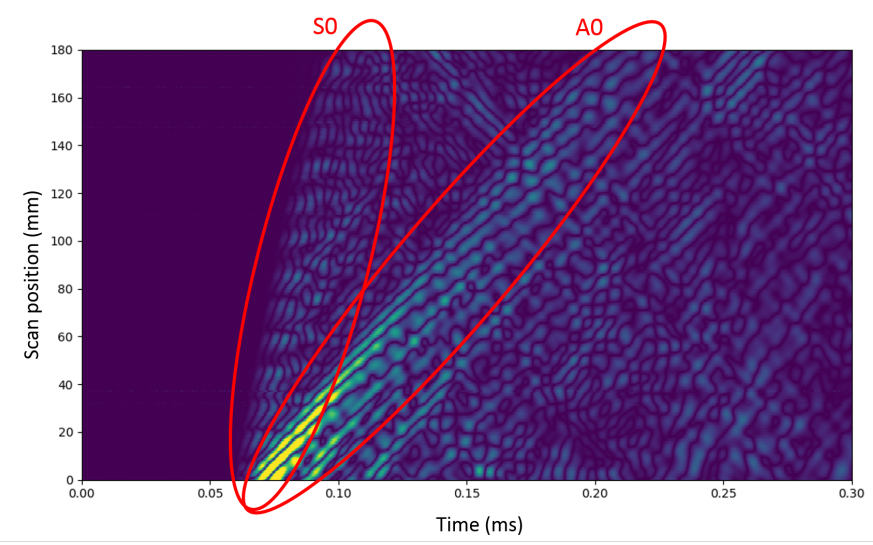

(a)

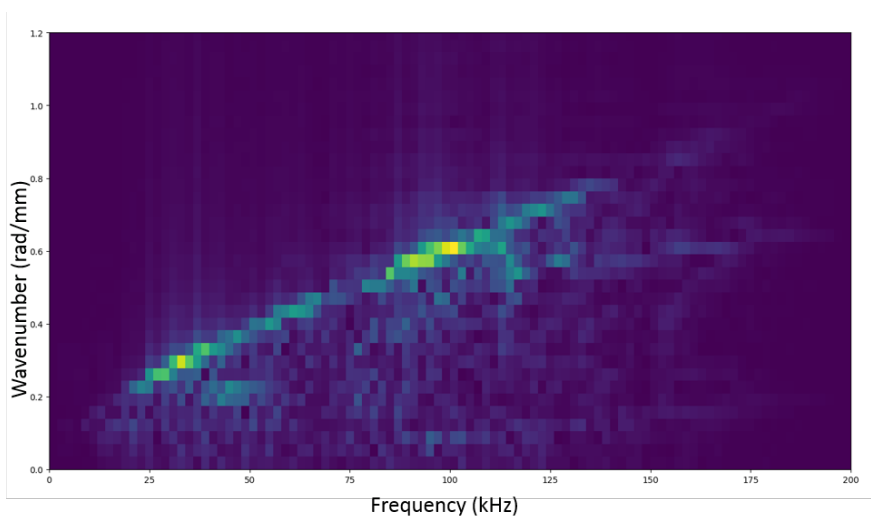

(b)

Figure 4: Type of results that can be obtained from measurements from the Laser Doppler Vibrometer performed on a textile composite plate.(a) Out-of-plane displacement magnitude $\left(U_{3}\right)$ measured by the scanning laser Doppler vibrometer as a function of space $x$ and time $t$. Two propagating modes (circled in red) can be observed (S0 and A0). (b) Dispersion curves obtained by 2D FFT of the experimental data. A0 can be observed.

\section{Textile composite modelling}

This section describes how the actual composites presented in Sec.2.1 are modeled. The mechanical characteristics of the components as well as the geometrical properties of the woven composites are sought (as shown in Fig.5). Their wave dispersion properties are then computed using the WFE/CMS methodology.

\subsection{Mechanical characteristics determination}

A yarn (or tow) is a bundle of aligned filaments (or fibres). Once the matrix is infused, the yarns are composed of fibres packed in a matrix of cured resin (see Fig.5). In reality the fibers are randomly distributed in the crosssectional view but the distribution will be considered regular and periodic as the prediction does not differ much [13]. To determine the yarn properties, a mechanical analysis based on idealised hexagonal single filament model can be performed [13, 14, 15] (analytical methods to obtain a good approximation of the mesoscale properties also exist [16] such as the Chamis method for example).

In the following subsection will be described the mechanical characteristics of the individual matrix and filaments materials composing a yarn in the first subsection. A second subsection will explain how the mechanical properties of a yarn are obtained from the ones from the matrix and filaments materials as shown in Fig.5.

\subsubsection{Microscale: Matrix and filaments materials}

The tensile modulus, the density and the average superficial density of the fabric are provided by the supplier, as well as for the resin's tensile modulus and density. These mechanical properties are displayed in Table A.3 in 

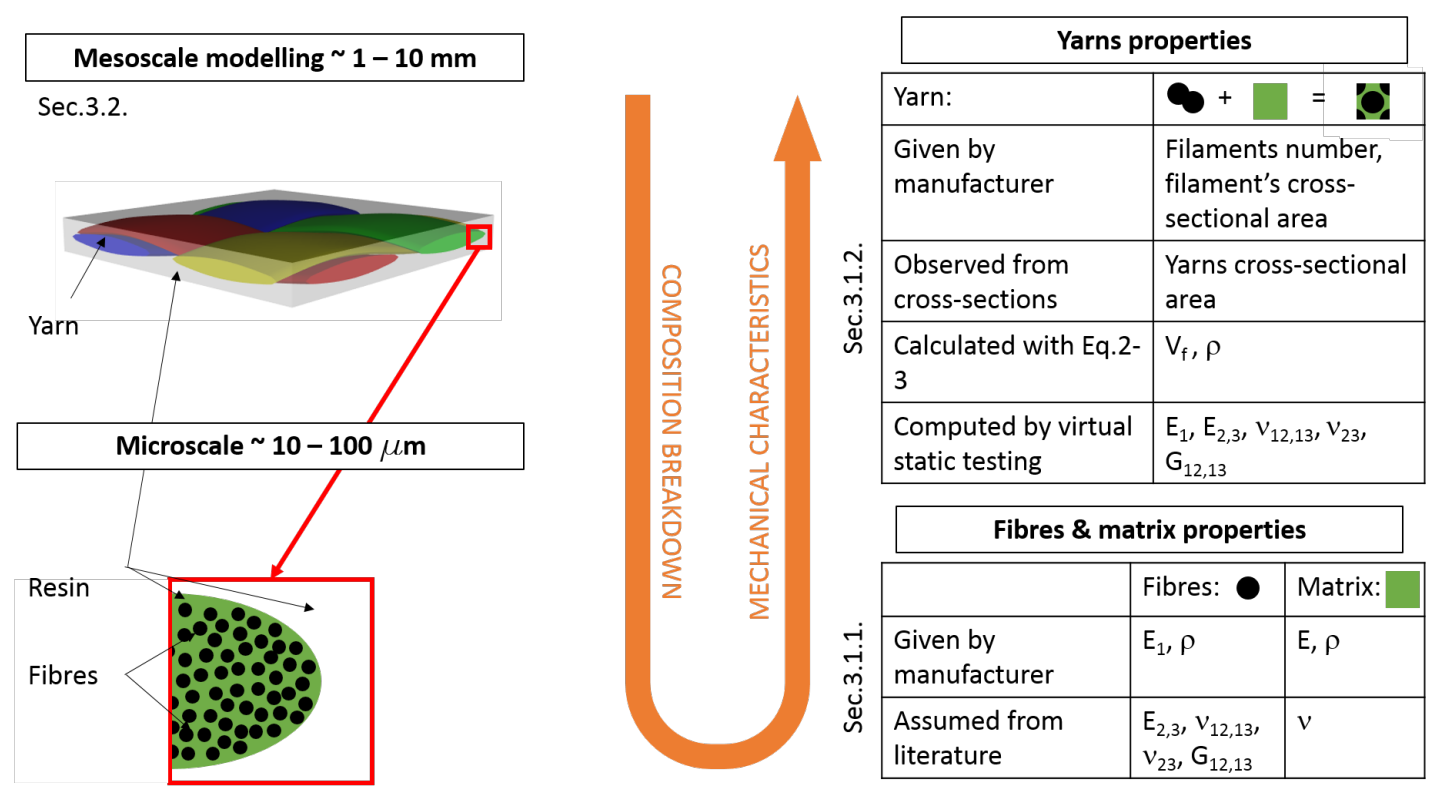

Figure 5: Determination of the mechanical properties with a multiscale approach. The textile is modelled at a mesoscopic scale. A microscale model is used to calculate the yarns effective mechanical properties. These properties are used in the mesoscale model alongside the matrix properties.

Appendix. While it is quite straightforward for the manufacturer to establish the static mechanical relations for an isotropic thermoplastic material such as epoxy, it is hard to provide the full set of transverse isotropic properties for a bundle of fibres (called tow or yarn). Indeed, tensile properties of the yarn, which are produced by gathering a large number of continuous filaments in a twisted form, are tested using a tensile testing machine [17] and thus only in the direction of the fibre. The transverse elastic modulus of the fibers can be measured by Raman spectroscopy or by nanoindentation for example but these are methods difficult to apply and the convergence is not assured [18, 19, 20]. This is not the only property that is neither given by the supplier, nor straightforward to measure. $G_{12,13}$ is measured via the torsional pendulum test $[19,21]$. For these reasons, some hypothesis need to be done for the other parameters. Firstly, the matrix material is considered isotropic, while the filaments and thus the yarns are considered transverse isotropic materials. For the matrix material, the missing engineering constant is the poisson ratio, while for the filaments, the transverse modulus $E_{2,3}$, the in plane shear modulus $G_{12,13}$ and the poisson ratios $v_{12,13}$ and $v_{23}$ are missing. All these missing properties will be selected from the literature (Tab.B.4 in AppendixB), and a sensitivity study will be performed to observe the impact of these constants on the final result.

From the literature, it is observed that $E_{2,3}$ ranges from 10 to $17 \mathrm{GPa}, G_{12,13}$ from 9 to $28 \mathrm{GPa}, v_{12,13}$ and $v_{23}$ from 0.2 to 0.25 for carbon fibers material, while $v$ is around 0.35 for epoxy resin material. For our study, $E_{2,3}$ is set to 15 $\mathrm{GPa}, G_{12,13}$ to $18 \mathrm{GPa}, v_{12,13}$ to 0.2 and $v_{23}$ to 0.25 for the filaments. As a filament is considered a transverse isotropic material, $G_{23}$ can be calculated using Eq.1. 


$$
G_{23}=\frac{E_{2,3}}{2\left(1+v_{23}\right)}
$$

$v$ is set to 0.35 for the matrix material.

\subsubsection{From microscale to mesoscale: Yarn mechanical properties}

In the previous section it is stated that the microstructure of a yarn is composed of numerous filaments and a matrix. The mesoscale model of a yarn consists in considering it as a single component made of an orthotropic material. Its properties are derived from the microscale model characteristics. For a yarn of aligned filaments, the fiber volume fraction $V_{f}^{\text {yarn }}$ is calculated using Eq.2 [9].

$$
V_{f}^{y a r n}=\frac{n_{f i l} A_{f i l}}{A_{\text {yarn }}}=\frac{n_{f i l} \pi R_{f i l}^{2}}{A_{\text {yarn }}}
$$

with $n_{f i l}$ the number of filaments in the yarn, $A_{y a r n}$ the cross-sectional area of a filament (also called fibre), $A_{y a r n}$ the cross-sectional area of the considered yarn.

The density is calculated using Eq.3.

$$
\rho_{y a r n}=\rho_{f} V_{f}^{\text {yarn }}+\rho_{m}\left(1-V_{f}^{\text {yarn }}\right)
$$

with $\rho_{f}$ the density of the fibre material, $\rho_{m}$ the density of the matrix material.

The homogenisation by static virtual testing from microscale (fibers \& resin) to mesoscale (yarn) gives us the properties for the different yarns of each material (Table C.5 in Appendix). Knowing the fiber volume fraction in each yarn, the total fiber volume fraction can be calculated as shown in Table 1. This is in adequation with the results from [9].

\begin{tabular}{|l|c|c|c|}
\hline Material & Material 1 & Material 2 & Material 3 \\
\hline Fibre volume fraction & 0.41 & 0.42 & 0.55 \\
\hline
\end{tabular}

Table 1: Fiber volume fraction in each of the three materials, in adequation with [9].

As the homogenisation is performed by static virtual testing, it implies that the microstructure has no effect on the dispersion properties of the mesostructure for low to middle range frequencies. A quick calculation is realized on a unit cell of a bundle made of an hexagonal arrangement of filaments to confirm the hypothesis. The unit cell is displayed in Fig.6.a.

The dispersion curves are computed for this unit cell using the method presented in [8] and compared with a unit cell made of an homogeneous material whose properties are acquired by virtual static testing homogenization. The results are displayed in Fig.6.b for one direction of propagation. It is observed that for low to middle frequency ranges, the internal architecture of the bundle has no effect on the dispersion properties. The hypothesis is considered valid for these applications. 


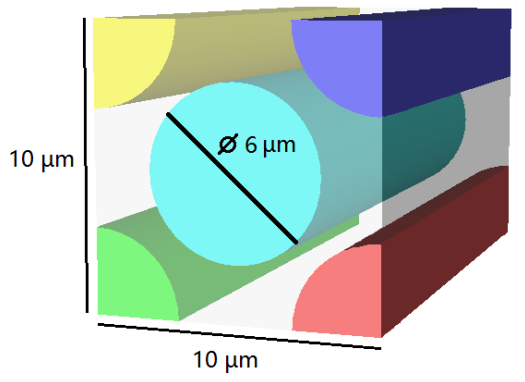

(a)

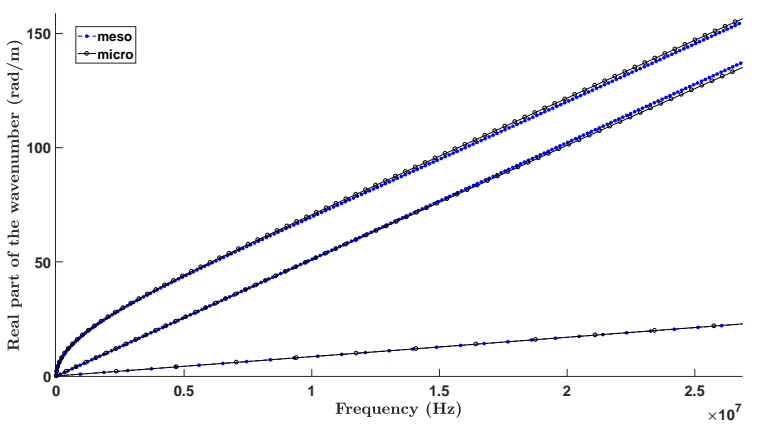

(b)

Figure 6: (a) Unit cell of the microscale structure of an hexagonal arrangement (b) Dispersion curves comparison between micro and mesoscale models of a unit cell of a fibre bundle

\subsubsection{Sensitivity analysis of the unknown microscale mechanical properties}

In Sec.3.1.1, the 'unknown' mechanical properties of the fibers and the matrix are chosen arbitrarily from the literature (see Table B.4 in Appendix). To ensure that the selection of the properties from the literature doesn't have a strong impact on the final results, a sensitivity study is performed on a simple 2D weave model composed of four yarns as displayed in Fig.5. Its fibre volume fraction is of 0.5, similarly to the studied experimental materials.

One by one all the parameters are set to a minimum and a maximum [min, max] (inspired from the literature) while the others remain the same (for the fiber material, $E_{2,3}$ are set to [10,20] GPa, $G_{12,13}$ are set to [8,28] GPa, $v_{12,13,23}$ are set to $[0.2,0.3]$ and for the matrix material $v_{\text {matrix }}$ is set to $\left.[0.3,0.35]\right)$, and the dispersion curves are computed using the WFEM/CMS methodology [8]. The value from the [min, max] couple that gives the lowest and highest wavenumbers function of the frequency for a mode are respectively indexed. All indexed parameters are then used to form respectively the lowest and highest dispersion curves possible (the 'worst case' scenarii). The lowest curves are obtained for the following set of parameters: $E_{2,3}=20 \mathrm{GPa}, G_{12,13}=28 \mathrm{GPa}, v_{12,13,23}=0.2$ and $v_{\text {matrix }}=0.35$. The higher curves are obtained for $E_{2,3}=10 \mathrm{GPa}, G_{12,13}=8 \mathrm{GPa}, v_{12,13,23}=0.3$ and $v_{\text {matrix }}=0.3$. The difference between the dispersion curves obtained with these two sets of parameters is of $4.15 \%$ for the flexural mode, $4.46 \%$ for the shear mode and of $4.38 \%$ for the pressure mode. These two 'worst case' scenarios do not provide considerable differences in the dispersion curves. Thus by choosing intermediate values for the missing parameters, the error is limited to a few percents.

\subsection{Geometric modelling of the yarns arrangement}

The first step to the methodology for computing the dispersion relations is the geometric modelling of a unit cell of the periodic woven composite of interest. The geometric modelling is done using a specialist pre-processor such as TexGen $[22,23]$. This open source software is developed by the composites research group at the University of Nottingham and is used for modelling the geometry of textile structure such as $2 \mathrm{D}$ or $3 \mathrm{D}$ woven textile. Indeed making 


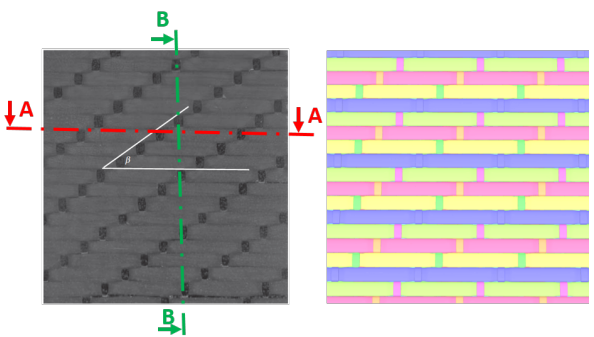

(a) Top view of Material 1

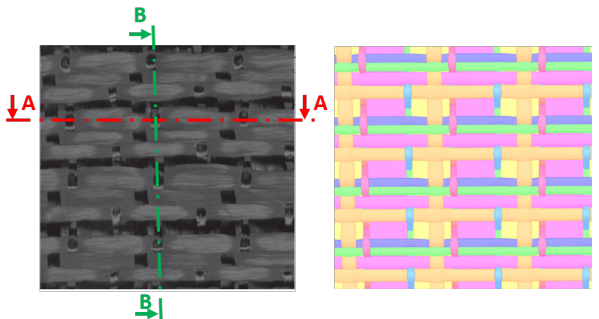

(c) Top view of Material 2

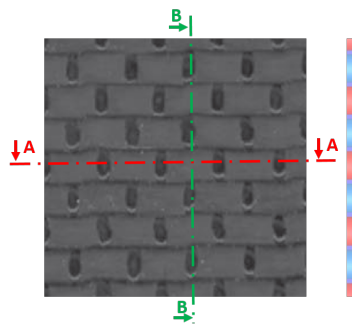

(e) Top view of Material 3

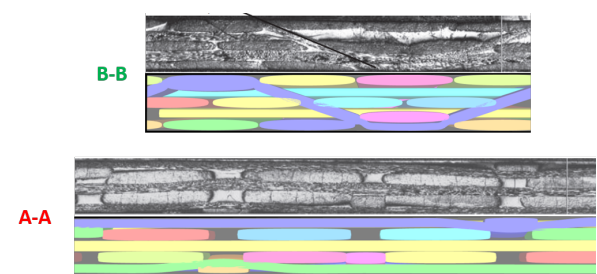

(b) Cross sections of Material 1

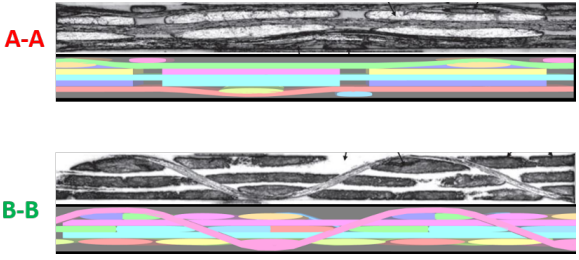

(d) Cross sections of Material 2

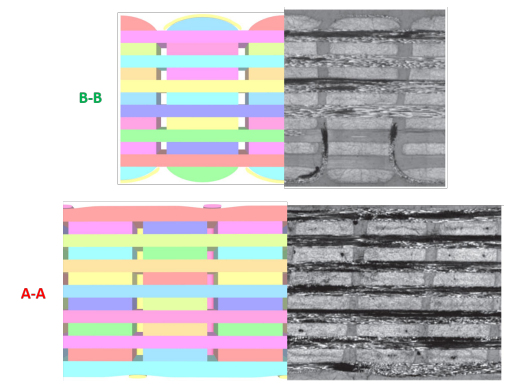

(f) Cross sections of Material 3

Figure 7: Left: photography of the actual composite [9] and the geometrical TexGen [28] model are compared - Right: micrographic scans of the actual composite [9] and the geometrical TexGen [28] model are compared

simplifying assumptions on the geometry of the yarns and on the structure itself can be tempting, but in reality the internal geometry of a textile composite is complex and the final shape of the pre-form is impacted by the chosen manufacturing process which can create nesting of the yarns for example [14, 15, 24, 25, 26]. For these reasons it is preferred to use observations from optical microscopy scans of the material as it helps for an accurate modelling of the geometric shapes $[26,27]$ as shown in Fig.7. In this figure can be observed the effort made toward an accurate geometric modelling of the textile composite as a good agreement between the photo/micro-graphies and the TexGen models screenshots is noted. The unit cells defining the geometry of the three studied textile composites are displayed in Fig.8.

A trade-off needs to be found between the sufficient number of elements to represent the geometrical features of the unit cell and a as low as possible number of degrees of freedom to facilitate the computation. This will be further 


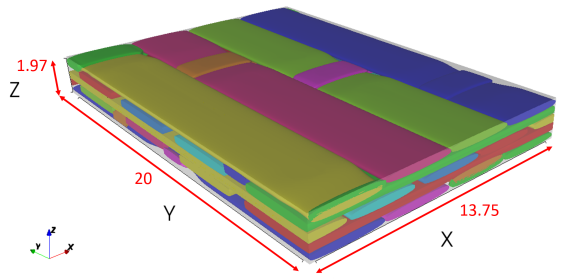

(a) Material 1

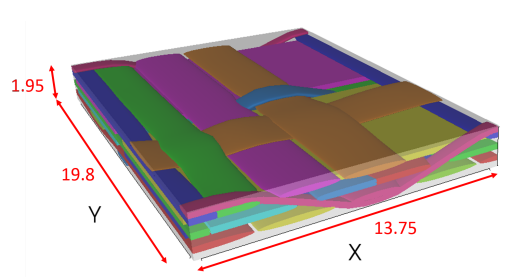

(b) Material 2

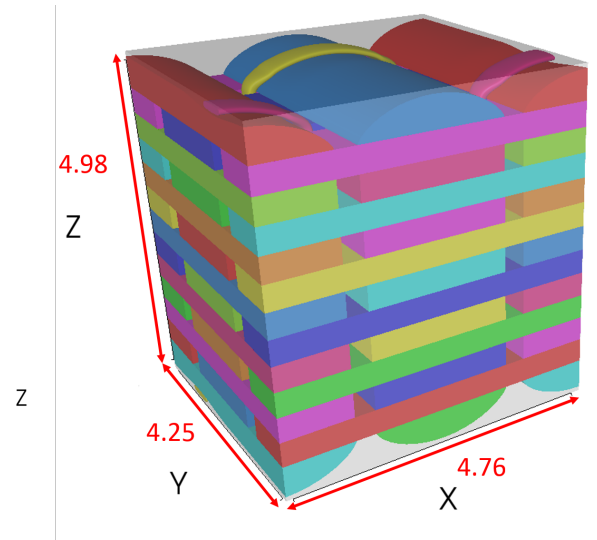

(c) Material 3

Figure 8: Unit cells of the three studied textile composites, (a) Material 1 is a classic 3D through-the-thickness angle interlock composite, (b) Material 2 is a less conventional 3D through-the-thickness angle interlock composite, (c) Material 3 is a classic 3D through-the-thickness orthogonal interlock composite - the dimensions are in mm

discussed in Sec.5.1.1. 


\subsection{Macroscale: Static virtual testing homogenisation of the whole unit cell}

In order to provide a comparison with the previously presented advanced methodology, some more traditional are presented in this section. These methodologies are based on the homogenisation of the mechanical parameters of a model to reduce the complexity of the problem and improve the speed of the calculations. There exist different ways to obtain the homogenized static mechanical properties of a 3D woven composite. The leading technique is to model the deformation using a FE model describing the internal geometry of the fabric [29]. This gives one orthotropic compliance matrix defining the whole material (Fig.9 method 1.). However considering that the material has a leading fibre orientation for each 'layer' (Fig.9 method 2.), an homogenization layer by layer might give better approached results as there are methods for obtaining the dispersion relations of laminates (WFEM, SAFE etc.) with each layer considered orthotropic. These two approaches will be used in Sec.4-5 for the sake of comparison.

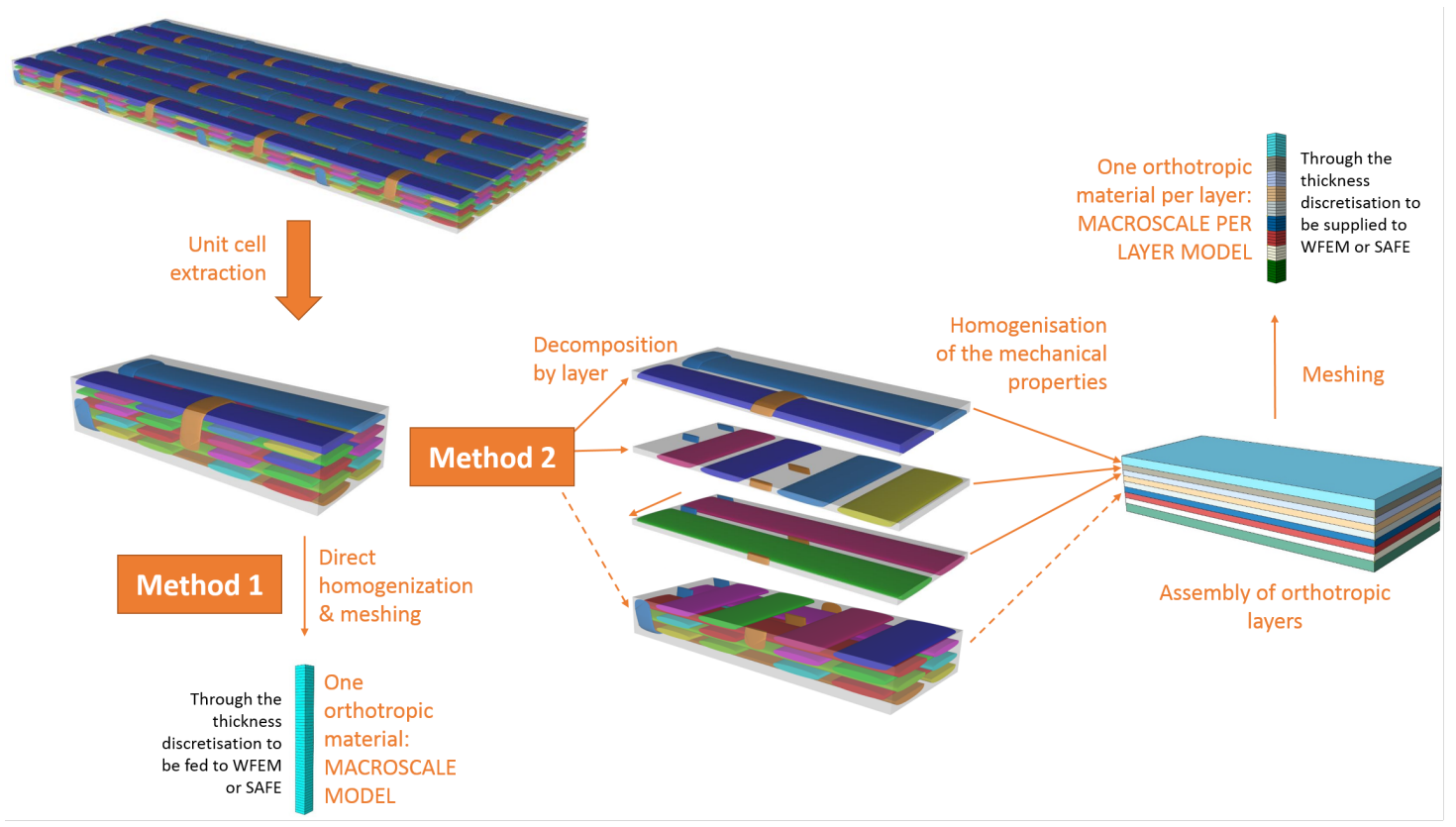

Figure 9: Unit cell homogenization: two methods are presented. One is the homogenization of the unit cell as a whole, which results in one set of orthotropic properties. The second considers an independant homogenization for each layer of the unit cell, resulting in as many sets of orthotropic properties as the number of layers.

\section{Wave propagation modelling}

In this paper, the two modelling approaches based on homogenization available in the literature and described in Sec.3.3, are compared to two numerical methods studying wave propagation properties taking into account the full textile geometry. These two numerical methods are presented in this section. The first one is the WFE/CMS method, short name for a method combining the wave finite element (WFE) method and the component mode synthesis (CMS) 
method. This modal approach is used to compute the dispersion relations of a complex textile composite while only a unit cell needs to be modeled. The method is thoroughly described in [8] but will be reminded briefly in Sec.4.1. The second method, which is a transient finite element analysis (Sec.4.2), is presented only to show the validity of the first method.

\subsection{WFE/CMS}

This section describes roughly the process of the WFE/CMS method but further details can be found in AppendixE for the WFE method and AppendixD for the CMS method.

Firstly, the nodal degrees of freedom (DoFs) of the unit cell are partitioned in the following way: bottom, top, left, right, left-bottom corner, right-bottom corner, left-top corner, right-top corner and internal DoFs, which gives:

$\mathbf{q}=\left\{\begin{array}{lllllllll}\mathbf{q}_{\mathbf{B}}^{\mathbf{T}} & \mathbf{q}_{\mathbf{T}}^{\mathbf{T}} & \mathbf{q}_{\mathbf{L}}^{\mathbf{T}} & \mathbf{q}_{\mathbf{R}}^{\mathbf{T}} & \mathbf{q}_{\mathbf{L B}}^{\mathbf{T}} & \mathbf{q}_{\mathbf{R B}}^{\mathbf{T}} & \mathbf{q}_{\mathbf{L T}}^{\mathbf{T}} & \mathbf{q}_{\mathbf{R T}}^{\mathbf{T}} & \mathbf{q}_{\mathbf{I}}^{\mathbf{T}}\end{array}\right\}^{T}$. The equation of motion of the unit cell can be written as

$$
\mathbb{M} \ddot{\mathbf{q}}(t)+\mathbb{C} \dot{\mathbf{q}}(t)+\mathbb{K} \mathbf{q}(t)=\mathbf{f}(t)
$$

Assuming time-harmonic behaviour leads to $\ddot{\mathbf{q}}=-\omega^{2} \mathbf{q}$, Eq. 4 can be rewritten as in Eq.5.

$$
\left[\mathbf{K}+\mathrm{i} \omega \mathbf{C}-\omega^{2} \mathbf{M}\right] \mathbf{q}=\mathbf{F}
$$

In order to reduce the complexity of the structural dynamic model, a Component Mode Synthesis (CMS) method is applied. It is mostly used to reduce the use of CPU time and memory.

The key to this method is the reduction of the relative DoFs (internal nodes), whereas the boundary DoFs are kept as physical coordinates [30]. In the fixed interface CMS, a set of 'fixed boundary modes', also called component modes are selected among a subset of the local modes of the unit cell when the boundary DoFs are fixed and no force is acting on the internal nodes. The reader is referred to Appendix for details on the method.

Once this reduction method has been applied, the periodic structure theory (PST) is employed (details on the method can be found in Appendix). The PST states that when a free wave travels along a waveguide, the displacements between two opposite boundary sides of a cell differ only by a propagation factor. The unit cell needs however to be meshed in a similar way on its opposite boundaries, so that continuity in displacements and forces equilibrium is respected [31], as shown in Fig.10.

In this case, (see Fig.10) the wave motion is in the $O x y$ plan, which gives Eq.E.1.

$$
\begin{aligned}
& \mathbf{q}_{\mathbf{R}}=\lambda_{x} \mathbf{q}_{\mathbf{L}} ; \quad \mathbf{q}_{\mathbf{T}}=\lambda_{y} \mathbf{q}_{\mathbf{B}} \\
& \mathbf{q}_{\mathbf{R B}}=\lambda_{x} \mathbf{q}_{\mathbf{L B}} ; \quad \mathbf{q}_{\mathbf{L T}}=\lambda_{y} \mathbf{q}_{\mathbf{L B}} ; \quad \mathbf{q}_{\mathbf{R T}}=\lambda_{x} \lambda_{y} \mathbf{q}_{\mathbf{L B}}
\end{aligned}
$$

$\lambda_{x}$ and $\lambda_{y}$ being respectively the propagation factors along the axis $x$ and $y$.

The propagation factors are calculated using Eq.7. 


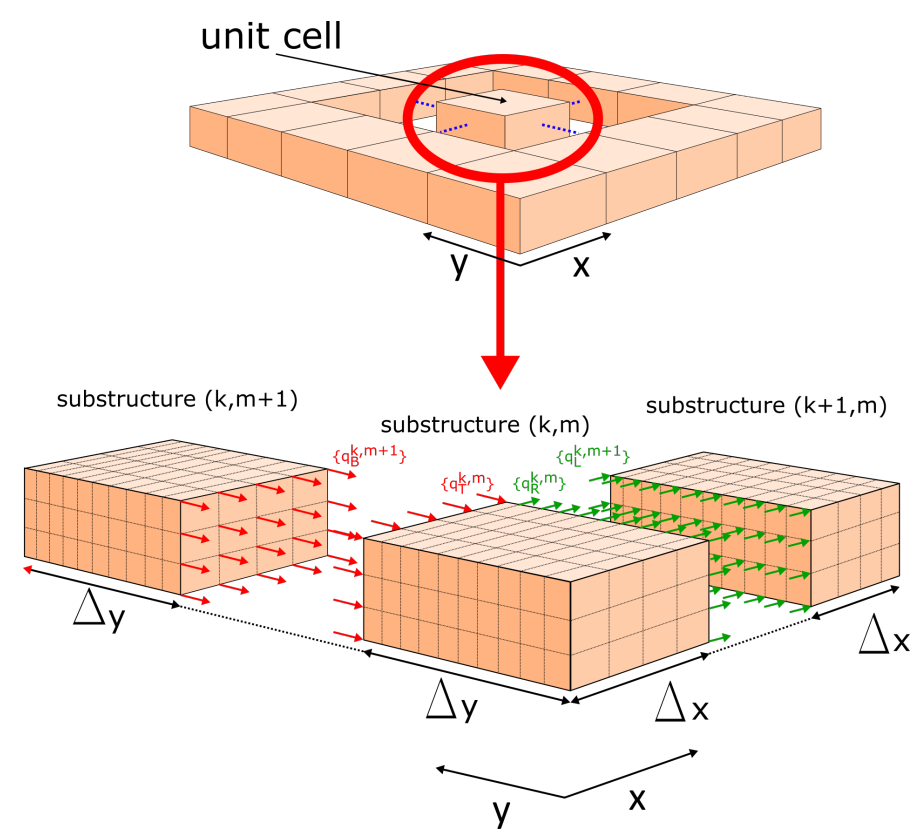

Figure 10: Periodic structure theory

$$
\lambda_{x}=\mathrm{e}^{-\mathrm{i} k_{x} \Delta_{x}} ; \quad \lambda_{y}=\mathrm{e}^{-\mathrm{i} k_{y} \Delta_{y}}
$$

and the direction of propagation $\theta$ is computed using Eq.8.

$$
k_{x}=k \cos (\theta) ; \quad k_{y}=k \sin (\theta)
$$

Combining the CMS reduction method with the periodicity relation, allows us to reduce drastically the number of unknown in the equations and thus the calculation time.

\subsection{Transient finite element analysis}

To ensure the numerical validity of the method, a transient finite element analysis of a textile composite is performed as another way to obtain its dispersion relations using Abaqus. As an example, a wave is generated in a beam made of Material 1 (see Fig.8.a). The beam is $20 \mathrm{~mm}$ wide (the length of a unit cell in the $y$-direction) and $2750 \mathrm{~mm}$ long (the length of 200 unit cells in the $x$-direction).

First of all, the displacement magnitude as a function of space and time (Fig.11.a) is plotted. Using the exact same beam model, only this time with homogenised mechanical properties determined as shown in Sec.3.3, the displacement magnitude as a function of space and time is plotted as well for comparison in Fig.11.b. It can be observed that the wavepackets displacement along the beam are more complex in the mesoscale model. Also, while the macroscale model captures accuratly some of the phenomenons observed in the mesoscale model such as the shear mode, it is yet not completely similar. 


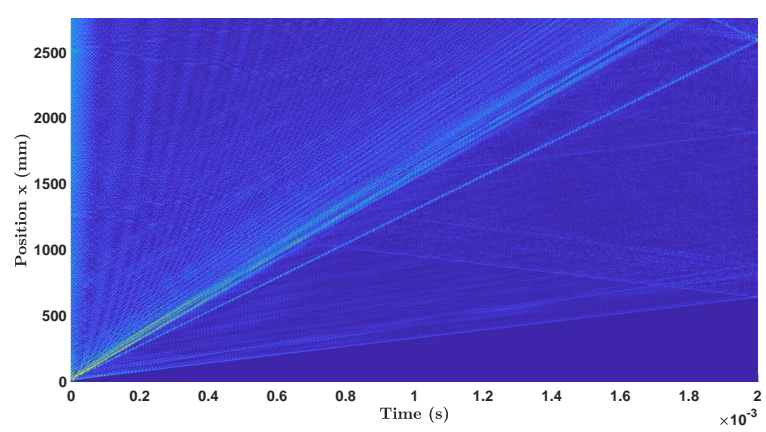

(a) Material 1 considered at a mesoscopic scale

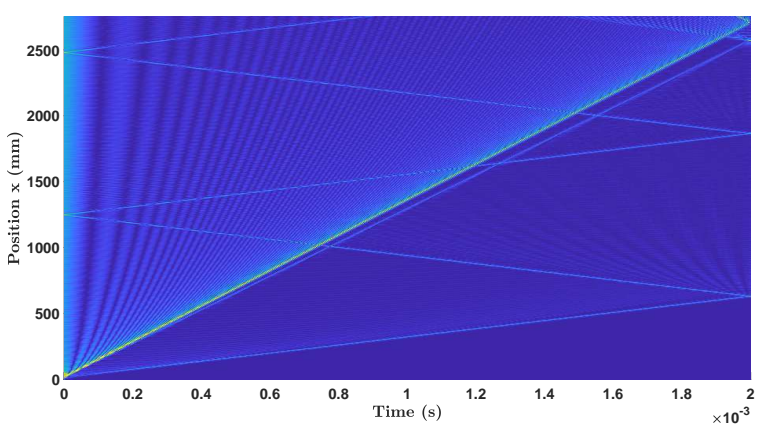

(b) Material 1 considered at a macroscopic scale (homogenised properties)

Figure 11: Displacements magnitude in the direction of propagation (U1) and out-of-plane (U3) captured by the FE model as a function of space $x$ and time $t$ in a beam made of Material 1

The dispersion characteristics are then computed and plotted in Fig.12 using the methodology presented in Sec.2.2 applied to numerical data. The WFE method is performed for the macroscale and macroscale per layer models as they are the quickest to compute. The results are displayed as well on the same figure. The macroscale model predicts the dispersion relations wrongly for most of the modes, while the dispersion characteristics predicted using the macroscale per layer model are close to the real dispersion characteristics of the mesoscale model for the five first modes. However it can be seen in the results obtained by the transient analysis that band-gaps are occurring for the flexural modes at around $60 \mathrm{kHz}$. These band-gaps are not predicted by the macroscale and macroscale per layer models. In conclusion, the results are seemingly different for the different studied scales, it can be deduced that the scale at which the modelling is performed does matter a lot for determining accurate dispersion relations.

Finally, in Fig.13 are plotted the results obtained through the WFE/CMS methodology along with the dispersion relations obtained with the transient analysis. Using Sec.4.1, the eigenvalue problem is solved with a given wavenumber formulation. $k$ is taken in the range $\left[0, \frac{\pi}{\Delta}\right]$. Indeed $\lambda=\mathrm{e}^{-\mathrm{i} k \Delta}$ is a periodic function, for that reason, the results are $\pi$ periodic and need to be considered only in the Brillouin Zone [32, 33, 34, 35]. It can be observed from this figure that the WFE/CMS methodology is very accurate to determine the dispersion relations as the agreement with the transient method is perfect. Also the dispersion curves exhibit bandgaps at the Brillouin Zone boundary $(k=2 \pi / \Delta)$ as enhanced by the yellow circle and as predicted in [8]. Another interesting feature is that some branches of the experimental curves are repeated in different Brillouin Zones as enhanced by the green dotted circles, this is due to the fact that Eq.7 gives the same results for $k$ or $k+2 n \pi / \Delta$. This phenomenon has been observed numerically and experimentally for periodic crystals in $[32,33,34,35]$. 


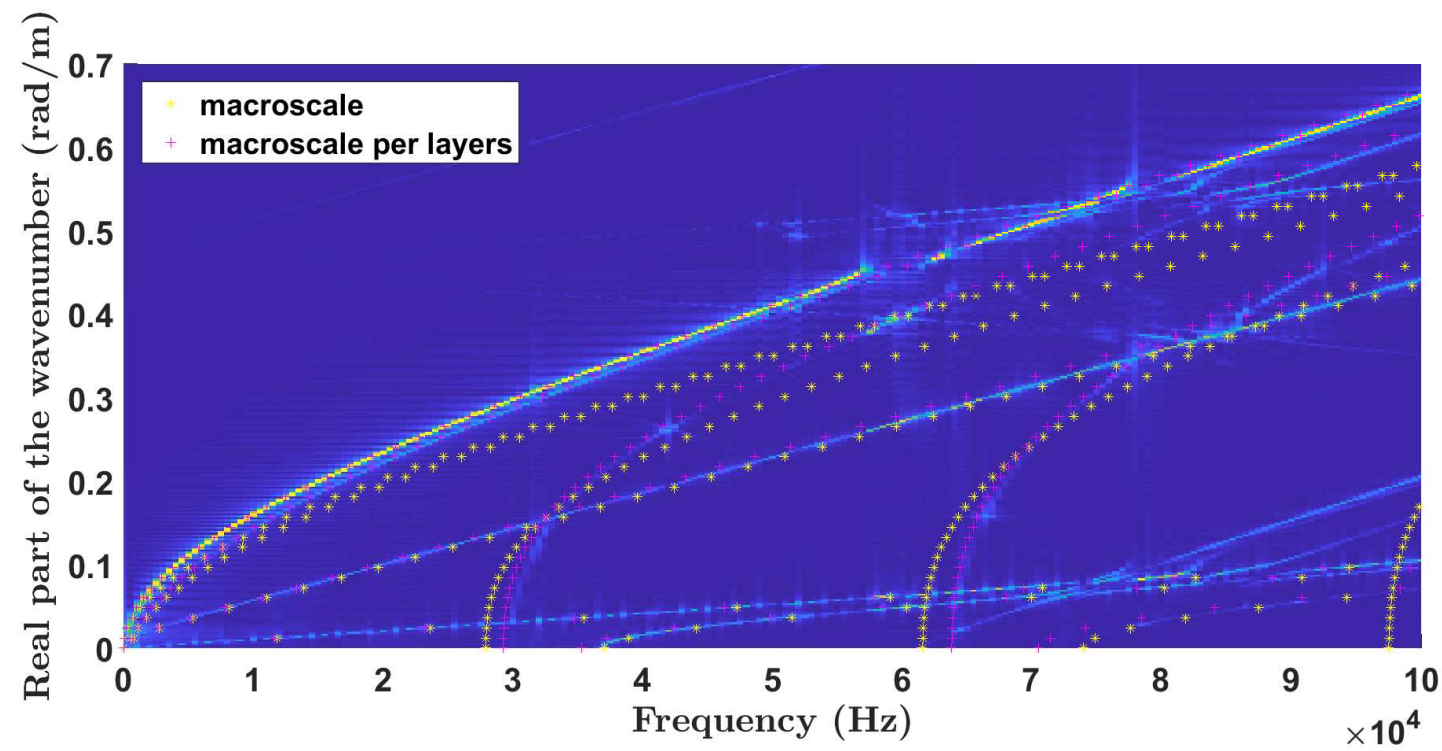

Figure 12: Dispersion relations in a beam made of Material 1 obtained by transient analysis (background) and by the WFE method with homogenised properties (see Fig.9), yellow '*' for macroscale and magenta '+' for macroscale per layer

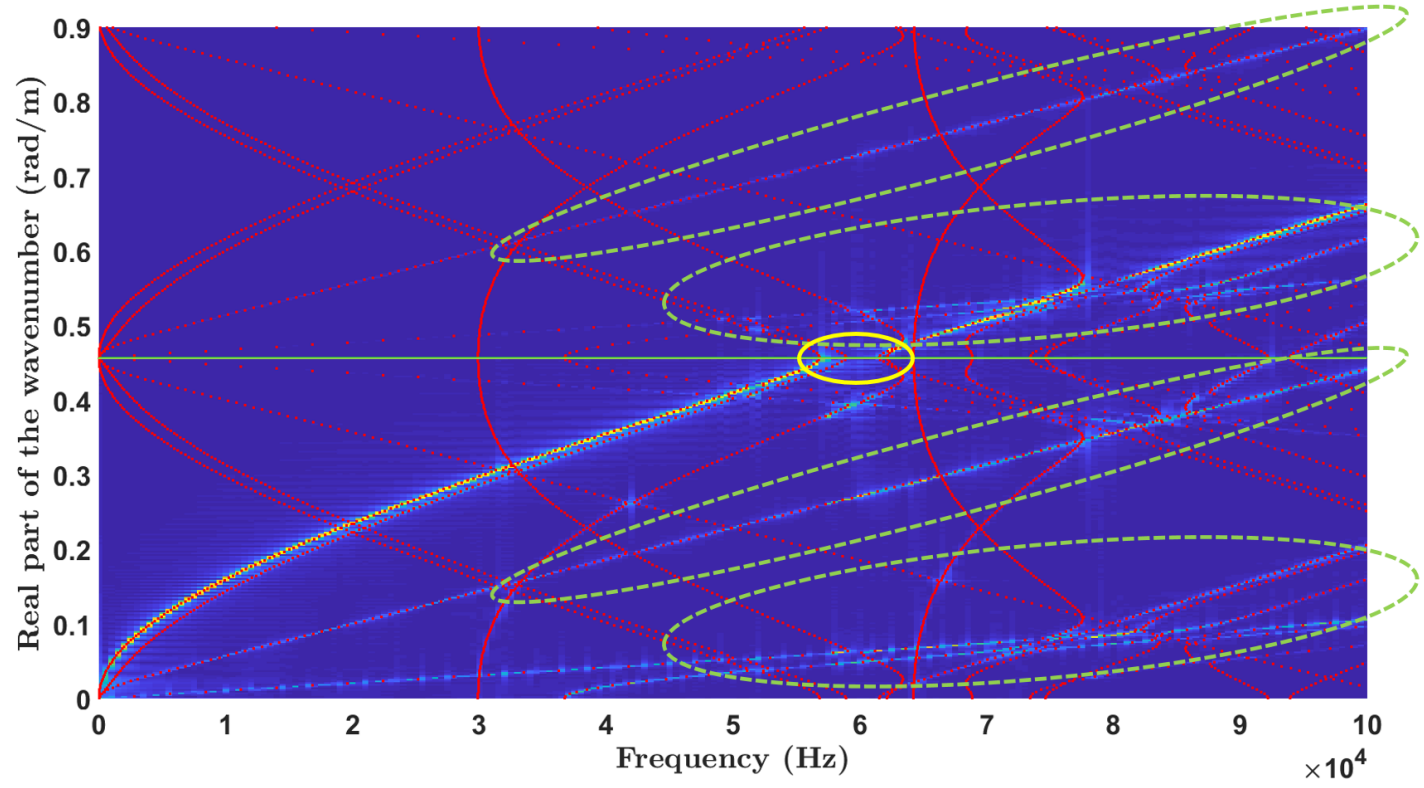

Figure 13: Dispersion relations in a beam made of Material 1 obtained by transient analysis (background) and by the WFE/CMS method (red dots). The green lines indicate the Brillouin zone boundaries $k=2 n \pi / \Delta$

\section{Results: comparison of the experimental and numerical results for the composite samples}

This section aims to compare the dispersion characteristics obtained experimentally (see Sec.2.2) and numerically as shown in Sec.4 for the three different studied textile composites. The results for each of the three materials will be 
presented in a separate subsection.

\subsection{Material 1: classic 3D through-the-thickness angle interlock composite}

The tested plate samples are made of Material 1 which is a classic 3D through-the-thickness angle interlock fabric impregnated in epoxy resin. The geometrical attributes of Material 1 can be visualized in Fig.7.a-.b. The plates measure 250x250x1.97 mm as presented in Sec.2.1. A unit cell of this composite as been modeled in Sec.3.2 and is shown in Fig.8.a.

\subsubsection{Mesh convergence study}

A quick convergence study is performed on the unit cell of Material 1 to ensure the validity of the mesh used. Five models, each with a different number of elements are created (40x22x10: 8800 elements; 50x30x12: 18000 elements; 60x40x15: 36000 elements; 70x50x20: 70000 elements; 80x60x25: 144000 elements). The convergence is obtained for the model 70x50x20 which always has less than 1\% of relative difference with the next model, containing twice as many elements, for any mode in the five studied directions of propagation.

\subsubsection{Experimental validation}

In Fig.14.a can be observed the experimental and numerical dispersion curves for the first modes propagating in the $\mathrm{x}$-direction. The picture resulting from the two-dimensional fast Fourier transform applied on the experimental data has a low resolution due to the length of the composite samples used $(250 \times 250 \mathrm{~mm})$ as explained in Sec.2.2. Three types of numerically obtained dispersion curves are also displayed. One results form the mesoscale methodology presented in Sec.3.2, while the last two result from the two macroscales modelling approaches as depicted in Fig.9.

It has to be noted that only the A0 and S0 can be observed on the dispersion curves obtained through experimental data. The first reason is that the studied frequency range is below the first cut-off frequency, so only the A0, S0 and SHO should be observed. The SHO mode is not measured by the laser vibrometer used for that study as it measures displacements in the direction normal to the surface ( $U_{3}$ direction). Thanks to the 15 degrees angle (relatively to the normal and around the y-axis) given to the laser beam $U_{1}$ can be captured and $\mathrm{S} 0$ can be observed.

The mesoscale model provides a really good agreement with the experimental results for both the S0 and A0 modes, while the A0 mode given by the macroscale model has a relative difference of $11.6 \%$ when compared to the experimental results (in the $x$-direction of propagation). It is however hard to conclude whether the mesoscale model or the macroscale per layer gives more accurate results as the picture resolution is low. Fig.14.b-d. and 16.a are similar to the previous one, only this time different directions of propagation are studied.

In directions other than $x$ and $y$ (Fig.14.b-.d), the S0 mode is not seen. This phenomenon can be explained by the energy focusing of Lamb waves. It is analog to the phonon focusing effect unveiled by Maris in [36], in which the energy flux is more intense in some directions. It can be predicted using the focusing (or Maris) factor [37, 38]. In Fig.15.a can be seen the slowness curve in Material 1 for the S0 mode for a propagation angle ranging from 0 to 90 


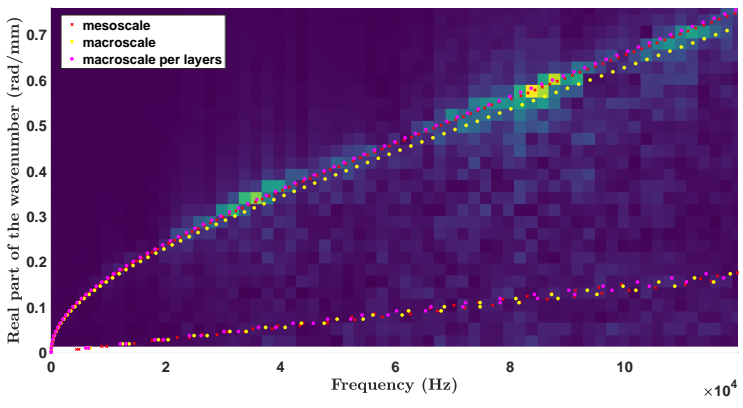

(a) Waves propagating in the $x$-direction

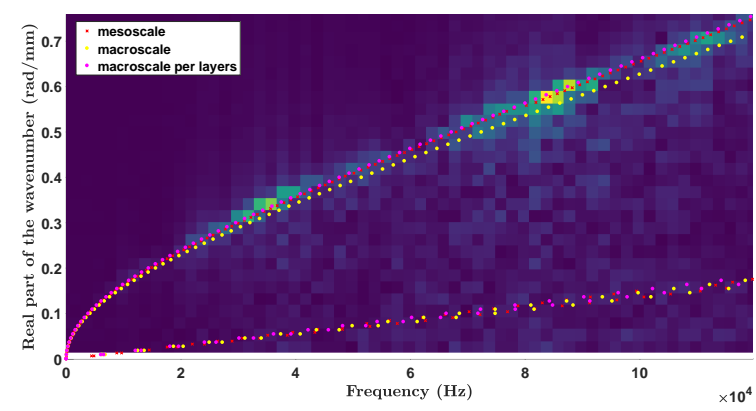

(c) Waves propagating at a 45 degrees angle to the $x$-direction

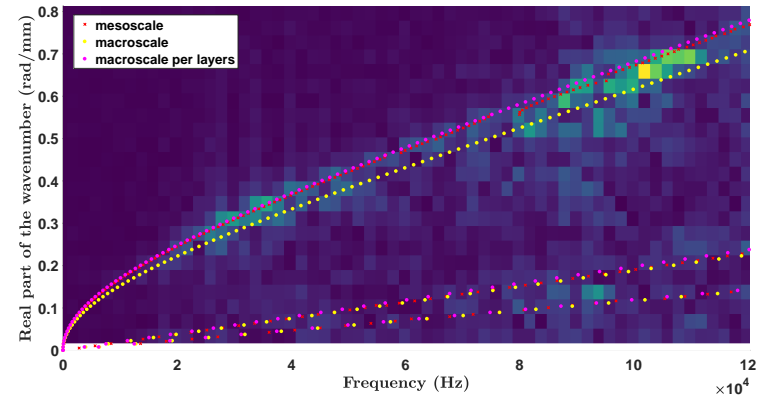

(b) Waves propagating at a 30 degrees angle to the $x$-direction

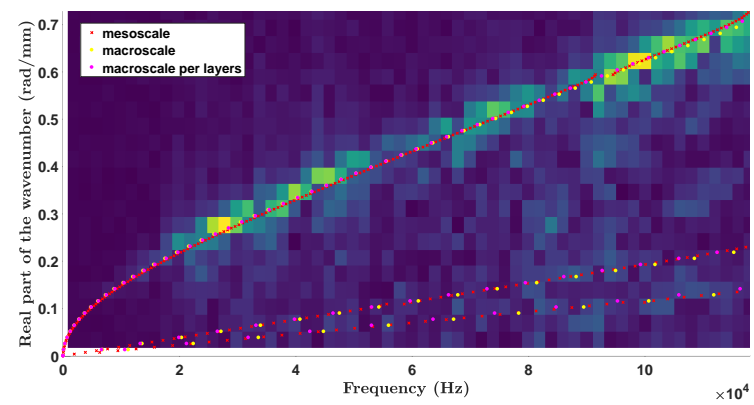

(d) Waves propagating at a 60 degrees angle to the $x$-direction

Figure 14: Dispersion relations for Material 1: 'background' experimentally obtained, ' $x$ ' obtained with the mesoscale method, 'magenta dots' obtained with the macroscale per layer method and 'yellow dots' obtained with the macroscale method 


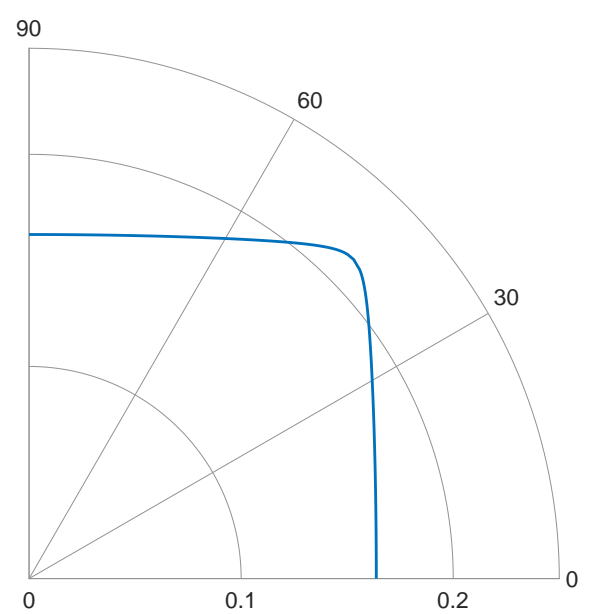

(a)

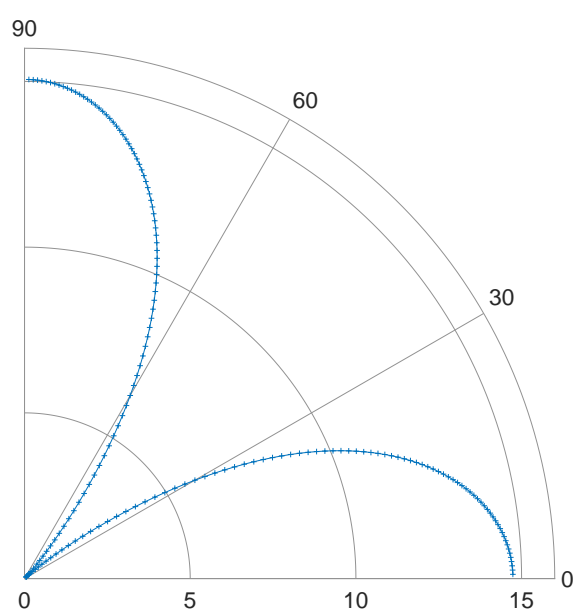

(b)

Figure 15: (a) Slowness curve in s. $\mathrm{km}^{-1}$ in Material 1 for S0 mode (b) Focusing factor A for the S0 mode

degrees. At low frequency-thickness products, the S0 mode is non dispersive and for that reason no frequency is given here. From the slowness curve, the focusing factor is computed and shown in Fig.15.b. It can be seen that the focusing is very intense in the main fibers directions ( $x$ and $y$-directions) and is very low in the other directions, especially at a 45 degrees direction of propagation. For each studied direction of propagation, the mesoscale and the macroscale per layer models are both in good agreement with the experimental results while the macroscale provides mostly wrong previsions for the A0 mode.

In Fig.16.a, which displays the experimental and numerical dispersion curves for the modes propagating in the y-direction can be observed another mode, different from the A0 and S0 modes we saw on the previous figures. Displaying the green lines indicating the Brillouin zone boundaries $k=n \pi / \Delta$, an hypothesis can be formulated. This material being periodic, we could be observing the same phenomenon as seen in Fig.13. The S0 mode is plotted again in white at $k=k+2 \pi / \Delta$ and it shows a good agreement with this 'third' experimentally observed mode. However, it is hard to explain why this mode does not appear in the other studied directions of propagation. In the numerical transient analysis (Fig.13) only a few modes are indeed repeated at $k=2 \pi / \Delta$, it is not clear why these ones are and not the others.

The discontinuities of the experimental dispersion curves are mainly justified by PZT frequency transduction effects and other experimental choices. The frequency of excitation is very broad, but centered around $100 \mathrm{kHz}$. For that reason, most captured magnitudes in the dispersion curves are present near that frequency. It is observed in the flexural mode in Fig.16.a a very low magnitude at $100 \mathrm{kHz}$, that is an effect of the frequency filtering operated by the PZT transducer (see Fig.16.b) in the $y$-direction. The high magnitude captured for the same mode at $37 \mathrm{kHz}$ is an effect of the frequency filtering again, it corresponds to the frequency at which the PZT transmits the most energy for that mode $[39,40]$. Even though this model is valid for predictions in isotropic media only, it provides some hints of 


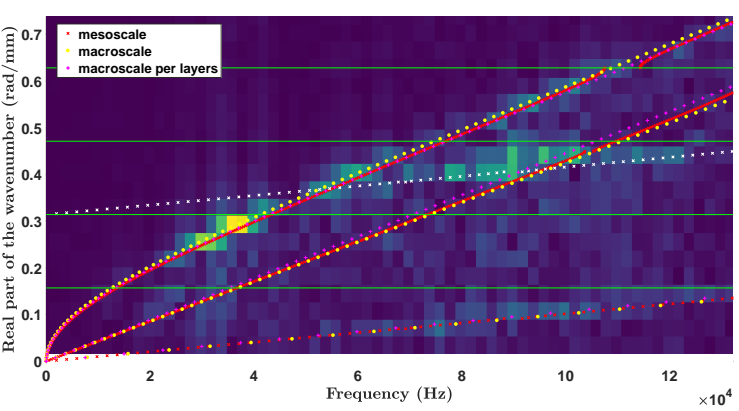

(a)

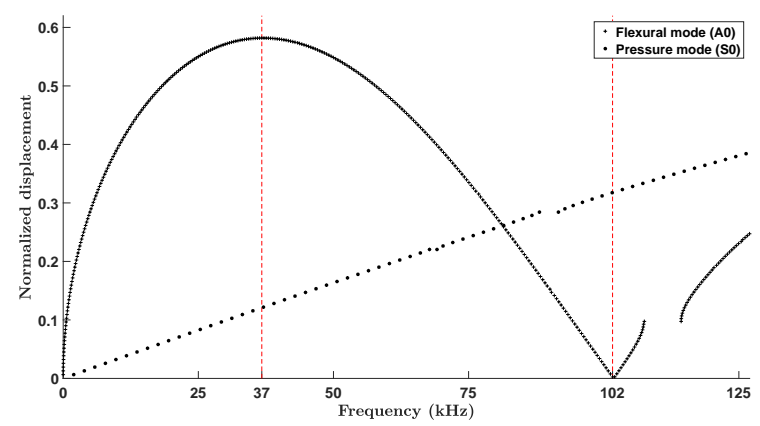

(b)

Figure 16: (a) Dispersion relations obtained for Material 1 for waves propagating in the $y$-direction; 'background' experimentally obtained, 'x' obtained with the mesoscale method, 'magenta dots' obtained with the macroscale per layer method and 'yellow dots' obtained with the macroscale method. The green lines indicate the Brillouin zone boundaries $k=n \pi / \Delta$ (b) Frequency filtering operated by the PZT transducer for the A0 and S0 modes in the y direction (PZT diameter of $13 \mathrm{~mm}$ )

explanation on the observed amplitude already. 


\subsection{Material 2: less conventional 3D through-the-thickness angle interlock composite}

The tested plate samples are made of Material 2 which is a less conventional 3D through-the-thickness angle interlock fabric impregnated in epoxy resin. The geometrical attributes of Material 2 can be visualized in Fig.7.c-.d. The plates measure 250x250x1.95 mm as presented in Sec.2.1. A unit cell of this composite has been modeled in Sec.3.2 and is shown in Fig.8.b.

The same study is performed on this material. This model's unit cell is discretized in 70x50x25 elements, totalizing 87500 elements. In Fig.17 can be observed the comparison between the experimental and numerical dispersion curves for different directions of propagation. The mesoscale results are in a good agreement with the experimental results.

For the first time, it is observed in Fig.17.c that the mesoscale and macroscale per layer models provide dispersion curves of the flexural mode that are significantly different. The mesoscale model gives results that are in good agreement with the experimental ones while the flexural modes given by the macroscale models are lower. 


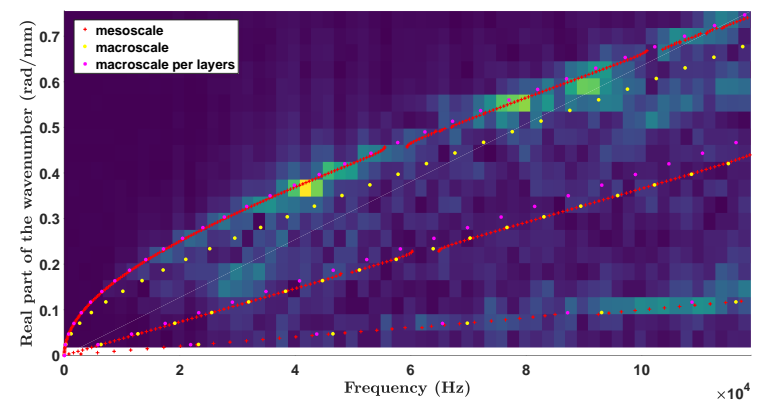

(a) Waves propagating in the $x$-direction

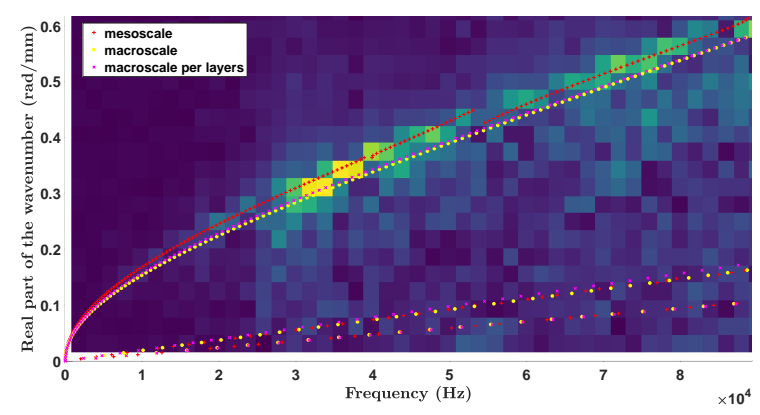

(c) Waves propagating at a 60 degrees angle to the $x$-direction

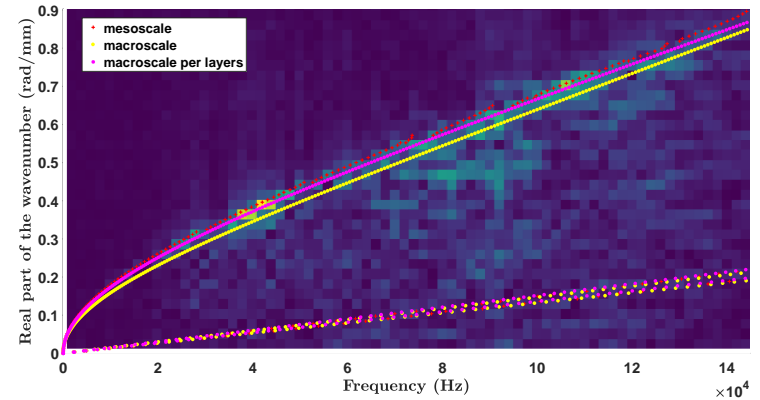

(b) Waves propagating at a 45 degrees angle to the $x$-direction

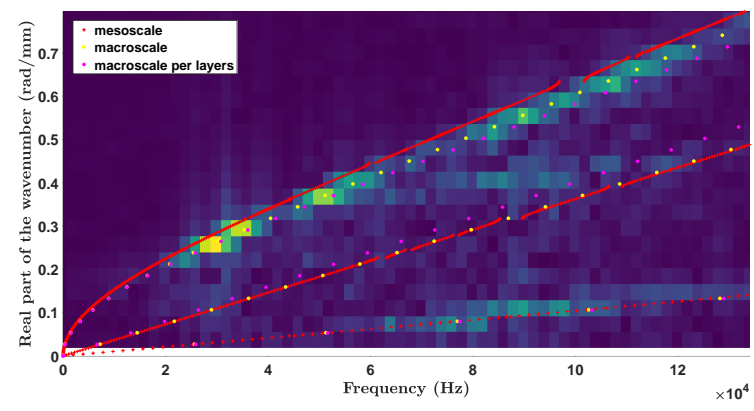

(d) [Waves propagating in the $y$-direction

Figure 17: Dispersion relations for Material 2: 'background' experimentally obtained, ' $x$ ' obtained with the mesoscale method, 'magenta dots' obtained with the macroscale per layer method and 'yellow dots' obtained with the macroscale method 


\subsection{Material 3: 3D through-the-thickness orthogonal interlock composite}

Here, the tested plate sample is made of Material 3 which is a classic 3D through-the-thickness orthogonal interlock fabric impregnated in epoxy resin. The geometrical attributes of Material 3 can be visualized in Fig.7.e-.f. The plate measures 250x250x4.98 mm as presented in Sec.2.1. A unit cell of this composite as been modeled in Sec.3.2 and is shown in Fig.8.c.

The same study is performed on the third material. This finite element model is composed of 35x35x60 elements, which means a total of 73500 elements and 237168 DoFs. It is observed that the mesoscale, the macroscale and the macroscale per layer models provide dispersion curves that are similar for the first three modes (see Fig.18). A significant difference can be observed between the mesoscale and macroscale per layer models for the shear mode in the $y$-direction (see Fig.18.d), unfortunately this mode could not be observed experimentally. Also the forth and fifth modes appear to be predicted differently with the macroscale model in Fig.18, but once again we were not able to observe these modes experimentally. This material did not allow us to conclude which model gives the best prediction.

The computation times for these models are displayed in Tab.2. The computation for the mesoscale models was run on the HPC of the University of Nottingham, it used 6 cores in parallel, each needing around 60 Go of RAM. The times shown are the elapsed times for calculating the dispersion relations in five different directions of propagation. To obtain the dispersion relations in another direction would be relatively costless timewise as the most consuming task is to project of the stiffness and mass matrices of the unit cell on the $\mathbf{B}$ basis obtained through the Craig-Bampton method to reduce the size of the set of internal DoFs (see Eq.D.5). The computation time is mainly driven by the number of boundary DoFs of the unit cell.

\begin{tabular}{|l|l|l|}
\hline & Elapsed time (with six cores) & CPU time (with one core) \\
\hline Material 1 mesoscale & 23 hours & around 3 days \\
\hline Material 2 mesoscale & 31 hours & around 4 days \\
\hline Material 3 mesoscale & 2 days & around 6 days \\
\hline Any material macroscale & - & $40-80$ seconds \\
\hline
\end{tabular}

Table 2: Computation time for all the models presented in this manuscript 


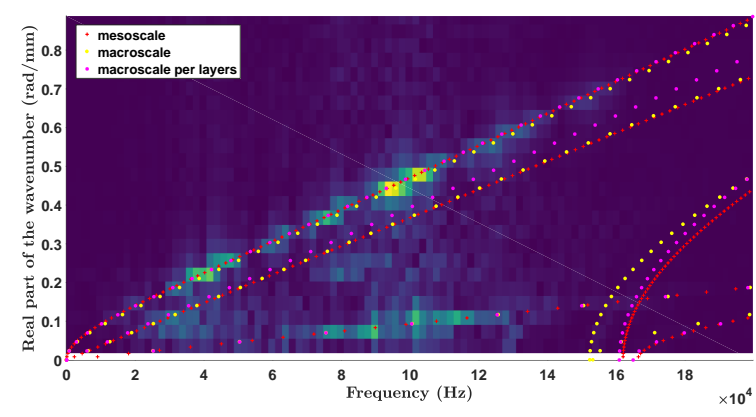

(a) Waves propagating in the $x$-direction

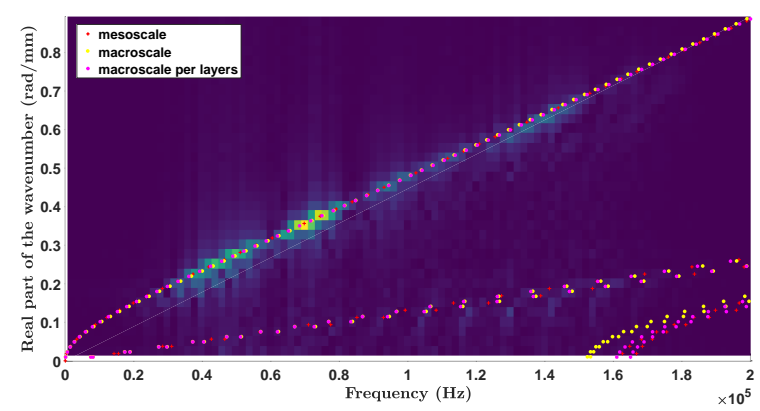

(c) Waves propagating at a 45 degrees angle to the $x$-direction

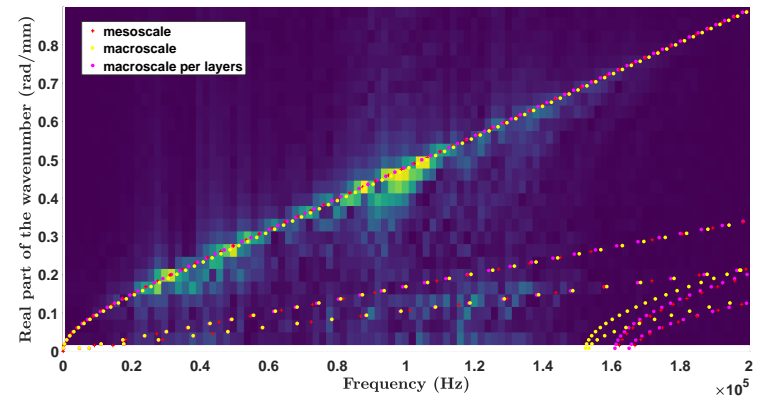

(b) Waves propagating at a 30 degrees angle to the $x$-direction

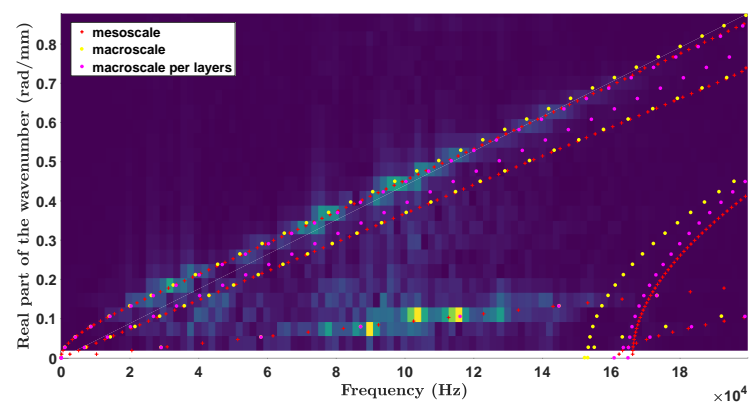

(d) Waves propagating in the $y$-direction

Figure 18: Dispersion relations for Material 3: 'background' experimentally obtained, ' $x$ ' obtained with the mesoscale method, 'magenta dots' obtained with the macroscale per layer method and 'yellow dots' obtained with the macroscale method 


\section{Conclusions}

In this paper a multiscale approach allowing for predicting the dispersion relations of complex woven composites is presented and compared with experimental results.

- Three levels of modelling are presented. The macroscale model is the coarsest and is equivalent to a model that could be created using only experimental static determination of the mechanical properties. This model is shown to be inaccurate in its predictions of the dispersion relations for the first and second presented materials.

- Numerically, the mesoscale model provides better predictions than the macroscale per layer model. However experimentally it is hard to tell them apart as we were not able to compare more than two modes (S0 and A0). It would have been possible to observe more modes at higher frequencies however the damping in the plates was too high. Another reason is the lack of resolution provided by the experimental results. It would be possible to have a higher resolution with larger plates as the inspected length defines the wavenumber resolution. This limitation comes from the manufacturing equipment used.

- It is shown that the computation times, even though relatively long for the mesoscale approach, are feasible. It should be noted that even though the macroscale per layer model is much faster to compute, the geometrical modelling step is the same for both models and it does take some time to complete as well. However in both cases, once this costly calculation is finished, the dispersion relations and their mode shapes are obtained for any direction of propagation at a minimal cost.

\section{Acknowledgments}

- This work is funded by the INNOVATIVE doctoral programme. The INNOVATIVE programme is partially funded by the Marie Curie Initial Training Networks (ITN) action (project number 665468) and partially by the Institute for Aerospace Technology (IAT) at the University of Nottingham.

- We are grateful for access to the University of Nottingham's Augusta HPC service.

- Special thanks go to the Composites Research Group members and especially to Louise Brown who gave us advices on TexGen, to Andreas Endruweit who gracefully provided us the dry fabrics and to the lab technicians Paul and Harry who helped us manufacture the composite samples.

- These experiments were conducted at the CEA List in France and we want to thank them for the help and opportunity provided on these, in particular Olivier Mesnil, Arnaud Recoquillay, Tom Druet, Alain Lhemery and Bastien Chapuis. 
[1] E. H. Glaessgen, C. M. Pastore, O. H. Griffin, and A. Birger, "Geometrical and finite element modelling of textile composites," Composites Part B: Engineering, vol. 27, no. 1, pp. 43-50, 1996.

[2] A. P. Mouritz, M. K. Bannister, P. J. Falzon, and K. H. Leong, "Review of applications for advanced three-dimensional fibre textile composites," Composites Part A: applied science and manufacturing, vol. 30, no. 12, pp. 1445-1461, 1999.

[3] A. D. Fergusson, Evaluating the mechanical behaviour of orthotropic 3D woven carbon fibre reinforced composites. Thesis, 2010.

[4] C. Willberg, S. Duczek, J. Vivar-Perez, and Z. Ahmad, "Simulation methods for guided wave-based structural health monitoring: a review," Applied Mechanics Reviews, vol. 67, no. 1, p. 010803, 2015.

[5] P. Tan, L. Tong, and G. P. Steven, "Modelling for predicting the mechanical properties of textile compositesa review," Composites Part A: Applied Science and Manufacturing, vol. 28, no. 11, pp. 903-922, 1997.

[6] L. Onal and S. Adanur, "Modeling of elastic, thermal, and strength/failure analysis of two-dimensional woven compositesa review," Applied Mechanics Reviews, vol. 60, no. 1, pp. 37-49, 2007.

[7] X. Lei, W. Rui, L. Yong, and L. Jin, "The effect of woven structures on the vibration characteristics of glass fabric/epoxy composite plates," Defence Science Journal, vol. 61, no. 5, 2011.

[8] V. Thierry, L. Brown, and D. Chronopoulos, "Multi-scale wave propagation modelling for two-dimensional periodic textile composites," Composites Part B: Engineering, 2018.

[9] A. Endruweit and A. C. Long, "Analysis of compressibility and permeability of selected 3d woven reinforcements," Journal of Composite Materials, vol. 44, no. 24, pp. 2833-2862, 2010.

[10] C. Xiaogang, L. W. Taylor, and L.-J. Tsai, "An overview on fabrication of three-dimensional woven textile preforms for composites," Textile Research Journal, vol. 81, no. 9, pp. 932-944, 2011.

[11] W. Sachse and Y. Pao, "On the determination of phase and group velocities of dispersive waves in solids," Journal of applied Physics, vol. 49, no. 8 , pp. 4320-4327, 1978 .

[12] D. Alleyne and P. Cawley, "A two-dimensional fourier transform method for the measurement of propagating multimode signals," The Journal of the Acoustical Society of America, vol. 89, no. 3, pp. 1159-1168, 1991.

[13] H. Yuanchen, J. Kyo Kook, and H. Sung Kyu, "Effects of fiber arrangement on mechanical behavior of unidirectional composites," Journal of Composite Materials, vol. 42, no. 18, pp. 1851-1871, 2008.

[14] M. Ansar, W. Xinwei, and Z. Chouwei, "Modeling strategies of 3d woven composites: a review," Composite structures, vol. 93, no. 8, pp. 1947-1963, 2011

[15] X. Zeng, L. P. Brown, A. Endruweit, M. Matveev, and A. C. Long, "Geometrical modelling of 3d woven reinforcements for polymer composites: Prediction of fabric permeability and composite mechanical properties," Composites Part A: Applied Science and Manufacturing, vol. 56, pp. 150-160, 2014.

[16] R. Younes, A. Hallal, F. Fardoun, and F. H. Chehade, Comparative review study on elastic properties modeling for unidirectional composite materials. intech, 2012.

[17] P. K. Mallick, Fiber-reinforced composites: materials, manufacturing, and design. CRC press, 2007.

[18] H. Miyagawa, T. Mase, C. Sato, E. Drown, L. T. Drzal, and K. Ikegami, "Comparison of experimental and theoretical transverse elastic modulus of carbon fibers," Carbon, vol. 44, no. 10, pp. 2002-2008, 2006.

[19] X. Ji, C. Wang, B. A. P. Francis, E. S. M. Chia, L. Zheng, J. Yang, S. C. Joshi, and Z. Chen, "Mechanical and interfacial properties characterisation of single carbon fibres for composite applications," Experimental Mechanics, vol. 55, no. 6, pp. 1057-1065, 2015.

[20] R. Maurin, P. Davies, N. Baral, and C. Baley, "Transverse properties of carbon fibres by nano-indentation and micro-mechanics," Applied Composite Materials, vol. 15, no. 2, pp. 61-73, 2008.

[21] J. F. Villeneuve and R. Naslain, "Shear moduli of carbon, si-c-o, si-c-ti-o and alumina single ceramic fibers as assessed by torsion pendulum tests," Composites Science and Technology, vol. 49, no. 2, pp. 191-203, 1993.

[22] M. Sherburn, Geometric and mechanical modelling of textiles. PhD thesis, University of Nottingham, 2007.

[23] H. Lin, L. P. Brown, and A. C. Long, "Modelling and simulating textile structures using texgen," Advanced Materials Research, vol. 331, 
pp. 44-47, 2011.

[24] S. D. Green, M. Y. Matveev, A. C. Long, D. Ivanov, and S. R. Hallett, "Mechanical modelling of 3d woven composites considering realistic unit cell geometry," Composite Structures, vol. 118, pp. 284-293, 2014.

[25] Q. T. Nguyen, E. Vidal-Sall, P. Boisse, C. H. Park, A. Saouab, J. Brard, and G. Hivet, "Mesoscopic scale analyses of textile composite reinforcement compaction," Composites Part B: Engineering, vol. 44, no. 1, pp. 231-241, 2013.

[26] R. D. B. Sevenois, D. Garoz, F. A. Gilabert, S. W. F. Spronk, S. Fonteyn, M. Heyndrickx, L. Pyl, D. Van Hemelrijck, J. Degrieck, and W. Van Paepegem, "Avoiding interpenetrations and the importance of nesting in analytic geometry construction for representative unit cells of woven composite laminates," Composites Science and Technology, vol. 136, pp. 119-132, 2016.

[27] N. Naouar, E. Vidal-Sall, J. Schneider, E. Maire, and P. Boisse, "Meso-scale fe analyses of textile composite reinforcement deformation based on X-ray computed tomography," Composite Structures, vol. 116, pp. 165-176, 2014.

[28] H. Lin, L. P. Brown, and A. C. Long, "Modelling and simulating textile structures using texgen," Advanced Materials Research, vol. 331, pp. 44-47, 2011.

[29] S. V. Lomov, D. S. Ivanov, G. Perie, and I. Verpoest, "Modelling 3d fabrics and 3d-reinforced composites: challenges and solutions," in 1ST WORLD CONFERENCE ON 3D FABRICS, pp. 9-11, Citeseer.

[30] L. G. J. Becker, “Cms methods for efficient damping prediction for structures with friction,” 2008.

[31] J. M. Mencik and M. N. Ichchou, "Multi-mode propagation and diffusion in structures through finite elements," European Journal of Mechanics - A/Solids, vol. 24, no. 5, pp. 877-898, 2005.

[32] P. Peier, H. Merbold, V. Pahinin, K. A. Nelson, and T. Feurer, "Imaging of thz waves in 2 d photonic crystal structures embedded in a slab waveguide," New journal of physics, vol. 12, no. 1, p. 013014, 2010.

[33] Z. K. Wang, V. L. Zhang, H. S. Lim, S. C. Ng, M. H. Kuok, S. Jain, and A. O. Adeyeye, "Nanostructured magnonic crystals with size-tunable bandgaps," ACS nano, vol. 4, no. 2, pp. 643-648, 2010.

[34] F. S. Ma, H. S. Lim, V. L. Zhang, S. C. Ng, and M. H. Kuok, "Magnonic band structure investigation of one-dimensional bi-component magnonic crystal waveguides," Nanoscale research letters, vol. 7, no. 1, p. 498, 2012.

[35] B. K. Ofori-Okai, P. Sivarajah, C. A. Werley, S. M. Teo, and K. A. Nelson, "Direct experimental visualization of waves and band structure in 2d photonic crystal slabs," New Journal of Physics, vol. 16, no. 5, p. 053003, 2014.

[36] H. J. Maris, "Enhancement of heat pulses in crystals due to elastic anisotropy," The Journal of the Acoustical Society of America, vol. 50, no. 3B, pp. 812-818, 1971.

[37] B. Chapuis, N. Terrien, and D. Royer, "Modeling and experimental investigations of lamb waves focusing in anisotropic plates," Journal of Physics: Conference Series, vol. 269, p. 012020, 2011.

[38] M. A. Torres-Arredondo, H. Jung, and C.-P. Fritzen, "A study of attenuation and acoustic energy anisotropy of lamb waves in multilayered anisotropic media for ndt and shm applications," in Proceedings of the 6th international workshop NDT in progress, Prague, Czech Republic: Brno University of Technology, pp. 313-325.

[39] V. Giurgiutiu, Structural health monitoring: with piezoelectric wafer active sensors. Elsevier, 2007.

[40] T. Druet, “Tomographie passive par ondes guidees pour des applications de controle sante integre,” 2017.

[41] M. Y. Matveev, Effect of variabilities on mechanical properties of textile composites. Thesis, 2015.

[42] M. Minus and S. Kumar, “The processing, properties, and structure of carbon fibers," Jom, vol. 57, no. 2, pp. 52-58, 2005.

[43] D. D. L. Chung, Carbon fiber composites. Elsevier, 2012.

[44] C. T. Sun and R. S. Vaidya, "Prediction of composite properties from a representative volume element," Composites Science and Technology, vol. 56, no. 2, pp. 171-179, 1996.

[45] I. P. Kumar, S. P. Kushwaha, P. Mohite, and S. Kamle, "Longitudinal shear modulus of single aramid, carbon and glass fibres by torsion pendulum tests," World Academy of Science, Engineering and Technology International Journal of Mechanical, Aerospace, Industrial and Mechatronics Engineering, vol. 8, 2014.

[46] C. L. Tsai and I. M. Daniel, "Determination of shear modulus of single fibers," Experimental Mechanics, vol. 39, no. 4, pp. 284-286, 1999. 
[47] X. Ji, A. M. Khatri, E. S. M. Chia, R. K. H. Cha, B. T. B. Yeo, S. C. Joshi, and Z. Chen, "Multi-scale simulation and finite-element-assisted computation of elastic properties of braided textile reinforced composites," Journal of Composite Materials, vol. 48, no. 8, pp. 931-949, 2013.

[48] L. Yu, D. Liu, K. Peng, and Y. He, "An improved torsion pendulum based on image processing for single fibers," Measurement Science and Technology, vol. 27, no. 7, p. 075601, 2016.

[49] C. Droz, C. Zhou, M. N. Ichchou, and J. P. Lain, "A hybrid wave-mode formulation for the vibro-acoustic analysis of 2d periodic structures," Journal of Sound and Vibration, vol. 363, pp. 285-302, 2016

[50] C. W. Zhou, J. P. Lain, M. N. Ichchou, and A. M. Zine, "Multi-scale modelling for two-dimensional periodic structures using a combined mode/wave based approach," Computers $\mathcal{E}$ Structures, vol. 154, pp. 145-162, 2015.

[51] E. Manconi and B. R. Mace, "Modelling wave propagation in two-dimensional structures using a wave/finite element technique," Institute of Sound and Vibration Research, 2007.

[52] E. Manconi and B. R. Mace, "Wave characterization of cylindrical and curved panels using a finite element method," J Acoust Soc Am, vol. 125 , no. 1 , pp. 154-63, 2009.

[53] C. Droz, J. P. Lain, M. N. Ichchou, and G. Inquit, "A reduced formulation for the free-wave propagation analysis in composite structures," Composite Structures, vol. 113, pp. 134-144, 2014. 
AppendixA. Mechanical properties datasheets

\begin{tabular}{|c|c|c|c|c|c|}
\hline MATERIAL 1 & Supplier & Product ref & Filament count & Tensile Modulus ( $G P a$ ) & Density $\left(\mathrm{g} / \mathrm{cm}^{3}\right)$ \\
\hline Warp tows & Tairylan & TC35-12K & $2 \times 12 \mathrm{~K}$ & 246 & 1.801 \\
\hline Weft tows & Tairylan & TC35-12K & $12 \mathrm{~K}$ & 246 & 1.801 \\
\hline Binder tows & Tairylan & TC33-6K & $6 \mathrm{~K}$ & 230 & 1.798 \\
\hline Resin & Easy Composites & IN2 & & 3.35 & 1.10 \\
\hline \multicolumn{6}{|l|}{ MATERIAL 2} \\
\hline Warp tows & Tairylan & TC35-12K & $4 \times 12 \mathrm{~K}$ & 246 & 1.801 \\
\hline Weft tows & Tairylan & TC35-12K & $12 \mathrm{~K}$ & 246 & 1.801 \\
\hline Warp interwoven tows & Tairylan & TC33-6K & $6 \mathrm{~K}$ & 230 & 1.798 \\
\hline Binder tows & Tairylan & TC33-6K & $6 \mathrm{~K}$ & 230 & 1.798 \\
\hline Resin & Gurit & Prime $20 \mathrm{LV}$ & & 3.5 & 1.144 \\
\hline \multicolumn{6}{|l|}{ MATERIAL 3} \\
\hline Warp tows & Teijin & HTS40 & $12 \mathrm{~K}$ & 240 & 1.77 \\
\hline Weft tows & Teijin & HTA40 & $2 \times 6 K$ & 240 & 1.77 \\
\hline Binder tows & Teijin & HTA40 & $1 \mathrm{~K}$ & 240 & 1.77 \\
\hline Resin & Easy Composites & IN2 & & 3.35 & 1.10 \\
\hline
\end{tabular}

Table A.3: Mechanical properties from the datasheets provided by the fibers manufacturers and resin providers 


\section{AppendixB. Mechanical properties in the literature}

\begin{tabular}{|c|c|c|c|c|c|c|}
\hline Lit ref & Product ref & $E_{1}$ & $E_{2,3}$ & $G_{12,13}$ & $v_{12,13}$ & $v_{23}$ \\
\hline [41] & Toray T300 & 230 & 15 & 13 & 0.24 & 0.24 \\
\hline [20] & Toray M40 & 294 & 15 & & & \\
\hline [20] & Toray M60 & 277 & 14 & & & \\
\hline [20] & Mitsubishi Pitch KL637 & 640 & 10.7 & & & \\
\hline [42] & & $200-500$ & $10-15$ & & & \\
\hline [13] & & 303 & 15.2 & 9.7 & 0.2 & 0.2 \\
\hline [43] & T300 & 230 & & 17 & & \\
\hline [43] & T400 & 226 & & 21.4 & & \\
\hline [43] & AS & 215 & & 21 & & \\
\hline [43] & M30 & 290 & & 17 & & \\
\hline [43] & M40 & 400 & & 15.8 & & \\
\hline [43] & M46 & 450 & & 14.8 & & \\
\hline [43] & $\mathrm{T} 800 \mathrm{H}$ & 290 & & 17 & & \\
\hline [43] & M-40J & 390 & & 17 & & \\
\hline [43] & M-46J & 450 & & 17 & & \\
\hline [24] & & 238 & 13 & 13 & 0.2 & 0.2 \\
\hline [44] & AS4 & 235 & 14 & 28 & 0.2 & 0.25 \\
\hline [21] & T300 & 231 & & 16 & & \\
\hline [45] & & & & 18.5 & & \\
\hline [46] & & & & 10.1 & & \\
\hline [47] & & 227.53 & 16.6 & 24.8 & 0.2 & 0.25 \\
\hline [48] & T300 & & & 15.8 & & \\
\hline [41] & Resin epoxy & 3.5 & 3.5 & & & 0.35 \\
\hline [13] & Resin epoxy & 3.31 & 3.31 & & & 0.35 \\
\hline
\end{tabular}

Table B.4: Fiber and resin mechanical properties obtained from the litterature 


\section{AppendixC. Mechanical properties of the yarns}

\begin{tabular}{|l|l|l|l|l|l|l|l|l|l|}
\hline & Yarn & $V_{f}^{\text {yarn }}$ & $E_{1}$ & $E_{2,3}$ & $G_{12,13}$ & $G_{23}$ & $v_{12,13}$ & $v_{23}$ & density \\
\hline Yarn - Mat1 & Warp & 0.643 & 159 & 7.76 & 4.83 & 3.40 & 0.247 & 0.434 & 1.55 \\
\hline & Weft & 0.421 & 105 & 5.79 & 2.72 & 2.33 & 0.281 & 0.481 & 1.39 \\
\hline & Binder & 0.610 & 141 & 7.32 & 4.29 & 3.20 & 0.253 & 0.446 & 1.53 \\
\hline Yarn - Mat2 & Warp & 0.705 & 173 & 8.84 & 6.27 & 3.78 & 0.239 & 0.405 & 1.61 \\
\hline & Warp tows & 0.364 & 85.1 & 5.62 & 2.50 & 2.17 & 0.291 & 0.485 & 1.38 \\
\hline & Weft & 0.683 & 169 & 8.52 & 5.76 & 3.68 & 0.242 & 0.415 & 1.59 \\
\hline & Weft compressed & 1.00 & 246 & 15.0 & 18.0 & 6.00 & 0.200 & 0.250 & 1.80 \\
\hline & Binder & 0.797 & 182 & 10.8 & 10.9 & 4.30 & 0.225 & 0.340 & 1.67 \\
\hline Yarn - Mat3 & Warp & 0.705 & 169 & 8.84 & 6.27 & 3.78 & 0.239 & 0.405 & 1.57 \\
\hline & Weft & 0.635 & 153 & 7.84 & 4.81 & 3.41 & 0.249 & 0.435 & 1.53 \\
\hline & Binder & 1.00 & 240 & 15.0 & 18.0 & 6.00 & 0.200 & 0.250 & 1.77 \\
\hline
\end{tabular}

Table C.5: Engineering constants for the yarns of the mesoscale model 


\section{AppendixD. Craig-Bampton Method}

The boundary nodal DOFs are written as $\mathbf{q}_{\mathbf{b}}=\left\{\begin{array}{llllllll}\mathbf{q}_{\mathbf{B}}^{\mathbf{T}} & \mathbf{q}_{\mathbf{T}}^{\mathbf{T}} & \mathbf{q}_{\mathbf{L}}^{\mathbf{T}} & \mathbf{q}_{\mathbf{R}}^{\mathbf{T}} & \mathbf{q}_{\mathbf{L B}}^{\mathbf{T}} & \mathbf{q}_{\mathbf{R B}}^{\mathbf{T}} & \mathbf{q}_{\mathbf{L T}}^{\mathbf{T}} & \mathbf{q}_{\mathbf{R T}}^{\mathbf{T}}\end{array}\right\}^{T}$. For free wave propagation, no external force acts on the internal nodes of the structure. This leads to $\mathbf{f}_{\mathbf{I}}=\mathbf{0}$. The equation of motion of the unit cell Eq. 5 becomes:

$$
\left(\left[\begin{array}{ll}
\mathbf{K}_{\mathbf{b b}} & \mathbf{K}_{\mathbf{b I}} \\
\mathbf{K}_{\mathbf{I b}} & \mathbf{K}_{\mathbf{I I}}
\end{array}\right]-\omega^{2}\left[\begin{array}{ll}
\mathbf{M}_{\mathbf{b b}} & \mathbf{M}_{\mathbf{b I}} \\
\mathbf{M}_{\mathbf{I b}} & \mathbf{M}_{\mathbf{I I}}
\end{array}\right]\right)\left\{\begin{array}{c}
\mathbf{q}_{\mathbf{b}} \\
\mathbf{q}_{\mathbf{I}}
\end{array}\right\}=\left\{\begin{array}{c}
\mathbf{f}_{\mathbf{b}} \\
\mathbf{0}
\end{array}\right\}
$$

In the fixed interface CMS, a set of 'fixed boundary modes', also called component modes $\boldsymbol{\Phi}_{\mathrm{C}}$ are selected among a subset of the local modes of the unit cell when the boundary DOFs are fixed and no force is acting on the internal nodes. Those local modes are the eigenvector $\boldsymbol{\Phi}_{\mathbf{I}}$ of Eq. D.2. The sus-mentioned subset is selected based on the lower resonance frequencies of the clamped model. That is why the modes are selected into the frequency range $\left[0,3 f_{\max }\right]$, with $f_{\max }$ being the maximum frequency of interest for the wave dispersion analysis $[49,50]$.

$$
\left[\mathbf{K}_{\mathbf{I I}}-\omega^{2} \mathbf{M}_{\mathbf{I I}}\right] \boldsymbol{\Phi}_{\mathbf{I}}=\mathbf{0}
$$

$\boldsymbol{\Phi}_{\mathbf{b}}$ is the static condensation and is calculated as follow

$$
\Phi_{\mathbf{b}}=\mathbf{K}_{\text {II }}^{-1} \mathbf{K}_{\mathbf{I b}}
$$

The projection matrix $\mathbf{B}$ can be determined as follow

$$
\left\{\begin{array}{c}
\mathbf{q}_{\mathbf{b}} \\
\mathbf{q}_{\mathrm{I}}
\end{array}\right\}=\left[\begin{array}{cc}
\mathbf{I} & \mathbf{0} \\
\boldsymbol{\Phi}_{\mathbf{b}} & \boldsymbol{\Phi}_{\mathbf{C}}
\end{array}\right]\left\{\begin{array}{c}
\mathbf{q}_{\mathbf{b}} \\
\mathbf{p}_{\Phi}
\end{array}\right\}=\mathbf{B}\left\{\begin{array}{c}
\mathbf{q}_{\mathbf{b}} \\
\mathbf{p}_{\Phi}
\end{array}\right\}
$$

The mass and stiffness matrix $\mathbf{M}$ and $\mathbf{K}$ can then be projected on the $\mathbf{B}$ basis

$$
\tilde{\mathbf{K}}=\mathbf{B}^{\mathrm{T}} \mathbf{K B}, \quad \tilde{\mathbf{M}}=\mathbf{B}^{\mathrm{T}} \mathbf{M B}
$$




\section{AppendixE. Wave and finite element method (WFEM)}

By using the Periodic Structure Theory (PST), the WFE method, allowing to find wave numbers of all the propagating waves by modelling only a period of the structure with standard FE, has been developed. This method has been extended by Manconi and Mace in [51] for free wave propagation in homogeneous structures in both $x$ and $y$ directions but whose properties may vary in the third direction through the thickness. The author applied this method in particular to isotropic, orthotropic and composite laminated plates and cylinders [51, 52].

In this case, the wave motion is in the Oxy plan, which gives

$$
\begin{aligned}
& \mathbf{q}_{\mathbf{R}}=\lambda_{x} \mathbf{q}_{\mathbf{L}} ; \quad \mathbf{q}_{\mathbf{T}}=\lambda_{y} \mathbf{q}_{\mathbf{B}} \\
& \mathbf{q}_{\mathbf{R B}}=\lambda_{x} \mathbf{q}_{\mathbf{L B}} ; \quad \mathbf{q}_{\mathbf{L T}}=\lambda_{y} \mathbf{q}_{\mathbf{L B}} ; \quad \mathbf{q}_{\mathbf{R T}}=\lambda_{x} \lambda_{y} \mathbf{q}_{\mathbf{L B}}
\end{aligned}
$$

And thus

$$
\left\{\begin{array}{c}
\mathbf{q}_{\mathbf{B}} \\
\mathbf{q}_{\mathbf{T}} \\
\mathbf{q}_{\mathbf{L}} \\
\mathbf{q}_{\mathbf{R}} \\
\mathbf{q}_{\mathbf{L B}} \\
\mathbf{q}_{\mathbf{R B}} \\
\mathbf{q}_{\mathbf{L T}} \\
\mathbf{q}_{\mathbf{R T}} \\
\mathbf{q}_{\mathbf{I}}
\end{array}\right\}=\left[\begin{array}{rrrr}
\mathbf{I} & \mathbf{0} & \mathbf{0} & \mathbf{0} \\
\mathbf{I} \lambda_{y} & \mathbf{0} & \mathbf{0} & \mathbf{0} \\
\mathbf{0} & \mathbf{I} & \mathbf{0} & \mathbf{0} \\
\mathbf{0} & \mathbf{I} \lambda_{x} & \mathbf{0} & \mathbf{0} \\
\mathbf{0} & \mathbf{0} & \mathbf{I} & \mathbf{0} \\
\mathbf{0} & \mathbf{0} & \mathbf{I} \lambda_{x} & \mathbf{0} \\
\mathbf{0} & \mathbf{0} & \mathbf{I} \lambda_{y} & \mathbf{0} \\
\mathbf{0} & \mathbf{0} & \mathbf{I} \lambda_{x} \lambda_{y} & \mathbf{0} \\
\mathbf{0} & \mathbf{0} & \mathbf{0} & \mathbf{I}
\end{array}\right]\left\{\begin{array}{c}
\mathbf{q}_{\mathbf{B}} \\
\mathbf{q}_{\mathbf{L}} \\
\mathbf{q}_{\mathbf{L B}} \\
\mathbf{q}_{\mathbf{I}}
\end{array}\right\}=\boldsymbol{\Lambda}_{\mathbf{R}}\left(\lambda_{x}, \lambda_{y}\right)\left\{\begin{array}{c}
\mathbf{q}_{\mathbf{B}} \\
\mathbf{q}_{\mathbf{L}} \\
\mathbf{q}_{\mathbf{L B}} \\
\mathbf{q}_{\mathbf{I}}
\end{array}\right\}
$$

Equilibrium at the right top corner nodes gives (subscript $b d$ stands for boundary):

$$
\boldsymbol{\Lambda}_{\mathbf{L}}\left(\lambda_{x}, \lambda_{y}\right)\left\{\begin{array}{c}
\mathbf{f}_{\mathbf{b d}} \\
\mathbf{0}
\end{array}\right\}=\mathbf{0}
$$

with $\boldsymbol{\Lambda}_{\mathbf{L}}\left(\lambda_{x}, \lambda_{y}\right)$ the conjugate transpose of $\boldsymbol{\Lambda}_{\mathbf{R}}\left(\lambda_{x}, \lambda_{y}\right)$

The following eigenvalue problem appears 


$$
\Lambda_{\mathbf{L}}\left(\mathbf{K}-\omega^{2} \mathbf{M}\right) \boldsymbol{\Lambda}_{\mathbf{R}}\left\{\begin{array}{c}
\mathbf{q}_{\mathbf{B}} \\
\mathbf{q}_{\mathbf{L}} \\
\mathbf{q}_{\mathbf{L B}} \\
\mathbf{q}_{\mathbf{I}}
\end{array}\right\}=0
$$

Combining the CMS reduction method Eq.D.4 with the periodicity relation Eq.E.2, we obtain

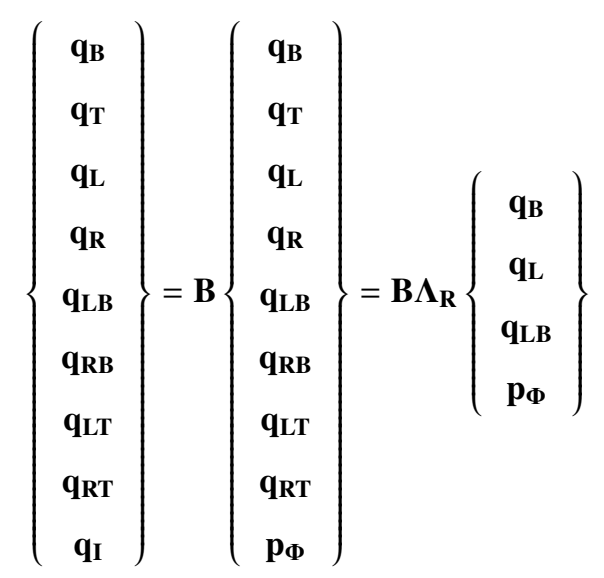

The eigenvalue problem can be solved with a given $\left(k_{x}, k_{y}\right)$ formulation [53]. 